Linköping Studies in Science and Technology

Dissertation No. 1560

\title{
Excitonic Effects and Energy Upconversion in Bulk and Nanostructured $\mathrm{ZnO}$
}

\author{
Shula Chen
}

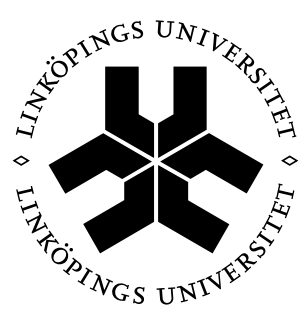

\section{Linköping University}

\author{
Division of Functional Electronic Materials \\ Department of Physics, Chemistry and Biology (IFM) \\ Linköping University, Sweden
}

Linköping 2014 
Cover figure (front): $5 \mathrm{~K}$ time-resolved photoluminescence (TRPL) of $\mathrm{ZnO}$ bulk single crystal from Tokyo Denpa Co. (top-right). The TRPL image was measured in a forward-transmission geometry i.e. laser excitation at back side and PL detection from front side. Also shown is an illustrative two-dimensional hexagonal lattice (bottom-left) with typical crystal defects: substitutional impurity, interstitial and vacancy.

Cover figure (back): $5 \mathrm{~K}$ three-dimensional TRPL image of $\mathrm{ZnO}$ bulk single crystal from Tokyo Denpa Co.. The TRPL images were recorded in two geometries: (1) back-scattering as marked by arrow ' $a$ ' and (2) forwardtransmission as marked by arrow ' $b$ '.

Copyright $\odot 2014$ Shula Chen, unless otherwise stated.

Excitonic Effects and Energy Upconversion in Bulk and Nanostructured $\mathrm{ZnO}$

ISBN: 978-91-7519-464-6

ISSN: 0345-7524

Linköping Studies in Science and Technology

Dissertation No. 1560

Printed by LiU-Tryck, Linköping, Sweden, 2014 


\section{Abstract}

Zinc Oxide ( $\mathrm{ZnO})$, a II-VI wurtzite semiconductor, has been drawing enormous research interest for decades as an electronic material for numerous applications. It has a wide and direct band gap of $3.37 \mathrm{eV}$ and a large exciton binding energy of $60 \mathrm{meV}$ that leads to intense free exciton (FX) emission at room temperature. As a result, $\mathrm{ZnO}$ is currently considered among the key materials for UV light emitting devices with tailored dimensionality and solid-state white lighting. Full exploration of $\mathrm{ZnO}$ for various applications requires detailed knowledge of its fundamental and material-related properties, which remains incomplete. The research work summarized in this thesis addresses a selection of open issues on optical properties of $\mathrm{ZnO}$ based on (but not limited to) detailed time-resolved photoluminescence (PL) and magneto-optical studies of various excitonic transitions as specified below.

Paper 1 and 2 analyze recombination dynamics of FX and donor bound excitons (DX) in bulk and tetrapod $\mathrm{ZnO}$ with the aim to evaluate contributions of radiative and non-radiative carrier recombination processes in the total carrier lifetime. We show that changes in relative contributions of these processes in "bulk" and near-surface areas are responsible for bi-exponential exciton decays typically observed in these materials. The radiative FX lifetime is found to be relatively long, i.e. $>1 \mathrm{~ns}$ at $77 \mathrm{~K}$ and $>14 \mathrm{~ns}$ at room temperature. In the case of DX, the radiative lifetime depends on exciton localization. Radiative recombination is concluded to dominate the exciton dynamics in "bulk regions" of high-quality materials. It leads to appearance of a slow component in the decays of no-phonon (NP) FX and DX lines, which also determines the dynamics of the longitudinal optical (LO) phonon-assisted and two-electronsatellite DX transitions. On the other hand, the fast component of the exciton decays is argued to be a result of surface recombination.

Paper 3 evaluates exciton-phonon coupling in bulk and tetrapod $\mathrm{ZnO}$. It is found that, in contrast to bulk $\mathrm{ZnO}$, the NP FX emission in $\mathrm{ZnO}$ tetrapods is weak as compared with the LO-phonon-assisted transitions. We show that the observed high intensity of the FX-1LO emission does not reflect enhanced excitonphonon coupling in nanostructured $\mathrm{ZnO}$. Instead, it is a result of stronger suppression of the NP FX emission in faceted regions of the tetrapods as revealed from spatially resolved cathodoluminescence (CL) studies. This is attributed to enhanced re-absorption due to multiple internal reflection, which become especially pronounced in the vicinity of the FX resonance.

Effects of exciton-photon coupling on light propagation through the $\mathrm{ZnO}$ media are studied in Paper 4 and 5. By employing the time-of-flight spectroscopy, in 
Paper 4, we demonstrate that the group velocity of laser pulses propagating through bulk $\mathrm{ZnO}$ can be slowed down to as low as $2044 \mathrm{~km} / \mathrm{s}$ when photon energies approach the optical absorption edge of the material. The magnitude of this decrease can be manipulated by changing light polarization. In Paper 5 we show that the observed slow-down is caused by the formation of free excitonpolaritons and is determined by their dispersion. On the other hand, contributions of DX polaritons become important only in the proximity of their corresponding resonances.

Excitonic effects can also be utilized to investigate fundamental properties and defect formation in $\mathrm{ZnO}$. In Paper 6, we employ DX to study magneto-optical properties of the $\mathrm{B}$ valence band (B-VB) states as well as dynamics of inter-VB energy relaxation. We show that PL decays of the emission involving the B-VB holes are faster than those of their counterparts involving the A-VB holes, which is interpreted as being due to energy relaxation of the holes assisted by acoustic phonons. Values of effective Landé g-factors for the B-VB holes are also accurately determined. In Paper 7, we uncover the origin of a new class of bound exciton lines detected within the near-band-edge region. Based on their magnetic behavior we show that these lines do not stem from DXs bound to either ionized or neutral donors but instead arise from excitons bound to isoelectronic center with a hole-attractive local potential.

In Paper 8, DX emissions are used to monitor energy upconversion in bulk and nanorod $\mathrm{ZnO}$. Based on excitation power-dependent PL measurements performed at different energies of excitation photons, the physical processes responsible for the upconversion are assigned to two-photon-absorption (TPA) via virtual states and two-step TPA (TS-TPA) via real states. In the former case the observed threshold energy for the TPA process is larger than half of that for one-photon absorption across the bandgap, which can be explained by the different selection rules between the involved optical transitions. It is also concluded that the TS-TPA process occurs via a defect/impurity with an energy level lying within 1.14-1.56 eV from one of the band edges, likely a zinc vacancy. 


\section{Populärvetenskaplig sammanfattning}

Zinkoxid (ZnO), II-VI wurtzit halvledare, har rönt ett enormt forskningsintresse i årtionden som ett elektroniskt material för talrika tillämpningar. Den har ett stort, direkt bandgap på $3.37 \mathrm{eV}$ och en stor exciton bindningsenergi av $60 \mathrm{MeV}$ som leder till intensiv emission via fria excitoner $(\mathrm{FX})$ vid rumstemperatur. Därför anses $\mathrm{ZnO}$ för närvarande vara bland de viktigaste materialen för UV-lysdioder med skräddarsydda dimensioner samt för vit belysning från fasta material. För fullt nyttjande av $\mathrm{ZnO}$ i olika tillämpningar krävs detaljerad kunskap om grundläggande och material-relaterade egenskaper som till viss del fortfarande är ofullständiga. Forskningsarbetet som sammanfattas i denna avhandling behandlar ett urval av de öppna frågor rörande optiska egenskaper hos $\mathrm{ZnO}$ och baseras på (men är inte begränsat till) detaljerade tidsupplösta och magneto-optiska fotoluminescens (PL) studier av olika excitoniska övergångar.

I artikel 1 och 2 analysers rekombinationsdynamiken av fria (FX) och donatorbundna excitoner (DX) i bulk tetrapod $\mathrm{ZnO}$ med syftet att utvärdera bidragen av utstrålande och icke-utstrålande rekombinationsprocesser i laddningsbärarnas totala livstid. Vi visar att förändringar i relativa bidragen av dessa processer $\mathrm{i}$ "bulk" och ytnära områden ansvarar för biexponentiell excitonavklingning som är typisk för dessa material. Den utstrålande FX livslängden visar sig vara relativt lång: över $1 \mathrm{~ns}$ vid $77 \mathrm{~K}$ och överstiger $14 \mathrm{~ns}$ vid rumstemperatur. I fallet med DX, beror den utstrålande livslängden på excitonens lokalisering. Utstrålande rekombination dominerar excitonens dynamik i "bulk regioner" av material med hög kvalitet. Det leder till uppkomsten av en långsam komponent i transienterna för de fononfria (NP) FX -och DX- linjerna och bestämmer också transienter av de längsgående optiska (LO) fononassisterade samt två-elektron-satellit DX övergångarna. $\AA$ andra sidan, är den snabba komponenten i exciton avklingningen ett resultat av ytrekombination.

I artikel 3 utvärderas exciton-fonon kopplingen i bulk och tetrapod ZnO. Man har funnit att i motsats till bulk $\mathrm{ZnO}$, är NP FX emision i $\mathrm{ZnO}$ tetrapoder svag i jämförelse med LO-fonon assisterade övergångar. Vi visar att den observerade höga intensiteten i FX-1LO emissionen inte återspeglar förbättrad exciton-fonon koppling i nanostrukturerade $\mathrm{ZnO}$. I stället är den ett resultat av undertryckande av NP FX emissionen och är som störst $\mathrm{i}$ de facetterade regionerna av tetrapoderna, något som avslöjades från rumsupplösta katodluminiscens (CL) 
studier. Detta tillskrivs ökad re-absorption på grund av flera interna reflektioner, vilket speciellt förstärks i närheten av FX resonansen.

Effekter av exciton-foton kopplingen på ljusutbredning genom $\mathrm{ZnO}$ medier studeras i artikeln 4 och 5. Genom att använda time-of-flight-spektroskopi, visar vi i artikel 4 att grupphastigheten av laserpulser som utbreder sig genom bulk $\mathrm{ZnO}$ kan saktas ned ända till $2044 \mathrm{~km} / \mathrm{s}$ när fotonenergier närmar sig materialets absorptionskant. Storleken av denna minskning kan manipuleras genom att ändra ljusets polarisering. I artikel 5 visar vi att den observerade nedgången orsakas av bildandet av fria exciton-polaritoner samt bestäms av deras spridning. $\AA$ andra sidan blir bidraget från DX polaritoner betydande endast i närheten av motsvarande resonanser.

Excitoniska effekter kan också användas för att undersöka fundamentala egenskaper och defektbildning i ZnO. I artikel 6, används DX för att studera magneto-optiska egenskaper av tillstånden i B-valensbandet (B-VB) samt dynamiken $\mathrm{i}$ inter-VB energirelaxationen. $\mathrm{Vi}$ visar att PL avklingningen som involverar B-VB hål är snabbare än deras motsvarighet som involverar A-VB hål, vilket tros bero på energirelaxation av hålen assisterad av akustiska fononer. Värden för effektiva Landé g faktorer för B-VB hål är också exakt utrönt. I artikel 7, avslöjar vi ursprunget av en ny klass BX linjer detekterade inom närabandkant-regionen. Baserat på deras magnetiska beteende visar vi att dessa linjer inte härrör från DXs bundna till varken joniserade eller neutrala donatorer utan istället uppstår från en exciton bunden till ett isoelektroniskt centrum med ett hålattraktiv potential.

Och slutligen i artikel 8, används DX PL för att granska energiuppkonverteringen i bulk $\mathrm{ZnO}$ samt $\mathrm{ZnO}$ nanostavar. Utifrån excitationseffekt-beroende PL mätningar utförda med olika excitationsenergier, är de processer som ansvarar för uppkonvertering tilldelade två-foton-absorption (TPA) via virtuella tillstånd samt två stegs TPA (TS-TPA) via verkliga tillstånd. Slutsatsen är att den sistnämnda processen sker via en defekt/förorening med en energinivå som ligger inom 1.14-1.56 eV från en av bandets kanter, sannolikt en zink vakans. En skarp energitröskel, som skiljer sig från den för motsvarande en-foton absorption, observeras för TPA-processen och förklaras i termer av urvalsregler för de inblandade optiska övergångarna. 


\section{Preface}

The work presented in this thesis was performed during the period of 2009-2013 in the Division of Functional Electronic Materials at Department of Physics, Chemistry and Biology (IFM), Linköping University, Sweden.

The thesis contains two parts. In the first part, a general overview of optical properties of $\mathrm{ZnO}$ is given together with a description of the utilized experimental methods. This part provides readers with background knowledge relevant to the research work presented in the thesis. The second part is a collection of the research articles listed below.

\section{Papers included in the thesis}

1. Long lifetime of free excitons in $\mathrm{ZnO}$ tetrapod structures.

S. K. Lee, S. L. Chen, H. Dong, L. Sun, Z. H. Chen, W. M. Chen, and I. A. Buyanova,

Applied Physics Letters 96, 083104 (2010)

2. Dynamics of donor bound excitons in $\mathrm{ZnO}$. S. L. Chen, W. M. Chen, and I. A. Buyanova, Applied Physics Letter 102, 121103 (2013)

3. On the origin of suppression of free exciton no-phonon emission in $\mathrm{ZnO}$ tetrapods.

S. L. Chen, S. K. Lee, W. M. Chen, H. Dong, L. Sun, Z. H, Chen, and I. A. Buyanova,

Applied Physics Letters 96, 033108 (2010)

4. Slowdown of light due to exciton-polariton propagation in $\mathrm{ZnO}$.

S. L. Chen, W. M. Chen, and I. A. Buyanova,

Physical Review B 83, 245212 (2011)

5. Long delays of light in $\mathrm{ZnO}$ caused by exciton-polariton propagation.

S. L. Chen, W. M. Chen, and I. A. Buyanova, 
Physica status solidi B 249, 1307 (2012)

6. Donor bound excitons involving a hole from the $\mathrm{B}$ valence band in $\mathrm{ZnO}$ : Time resolved and magneto-photoluminescence studies.

S. L. Chen, W. M. Chen, and I. A. Buyanova, Applied Physics Letters 99, 091909 (2011)

7. Zeeman splitting and dynamics of an isoelectronic bound exciton near the band edge of $\mathrm{ZnO}$.

S. L. Chen, W. M. Chen, and I. A. Buyanova,

Physical Review B 86, 235205 (2012)

8. Efficient upconvertion of photoluminescence via two-photon-absorption in bulk and nanorod $\mathrm{ZnO}$.

S. L. Chen, J. Stehr, N. K. Reddy, C. W. Tu, W. M. Chen, and I. A.

Buyanova,

Applied Physics B 108, 919 (2012)

\section{My contribution to these publications}

Paper 1. I have participated in measurements, performed data analysis and together with my co-authors interpreted the data.

Papers 2-7. I have performed all experimental work and data analysis, interpreted data together with my co-authors and wrote the first version of the manuscript.

Paper 8. I have performed all optical measurements and analyzed the corresponding data, interpreted data together with my co-authors and wrote the first version of the manuscript. 


\section{Papers not included in the thesis}

1. Optical properties of GaP/GaNP core/shell nanowires: a temperaturedependent study.

A. Dobrovolsky, S. L. Chen, Y. J. Kuang, S. Sukrittanon, C. W. Tu, W.

M. Chen, and I. A. Buyanova,

Nanoscale Research Letters 8, 239 (2013)

2. Defect properties of $\mathrm{ZnO}$ nanowires revealed from an optically detected magnetic resonance study.

J. Stehr, S. L. Chen, S. Filippov, M. Devika, N. Reddy, C. W. Tu, W. M.

Chen, and I. A. Buyanova,

Nanotechnology 24, 015701 (2013)

3. Evidence for coupling between exciton emissions and surface plasmon in Ni-coated ZnO nanowires.

Q. Ren, S. Filippov, S. L. Chen, M. Devika, N. Reddy, C. W. Tu, W. M.

Chen, and I. A. Buyanova,

Nanotechnology 23, 425201 (2012)

4. Mechanism for radiative recombination and defect properties of GaP/GaNP core/shell nanowires.

A. Dobrovolsky, J. Stehr, S. L. Chen, Y. J. Kuang, S. Sukrittanon, C. W. Tu, W. M. Chen, and I.A. Buyanova, Applied Physics Letters 101, 1631061 (2012)

\section{Conference contributions}

1. Isoelectronic bound excitons in $\mathrm{ZnO}$ : Time-resolved and Magneto-PL study.

S. L. Chen, W. M. Chen, and I. A. Buyanova,

The $27^{\text {th }}$ International Conference on Defects in Semiconductors, July 21-26, 2013, Bologna, Italy. 
2. Efficient upconvertion of photoluminescence via two-photon-absorption in bulk and nanorod $\mathrm{ZnO}$.

S. L. Chen, W. M. Chen, N. Koteeswara Reddy, C. W. Tu and I. A. Buyanova,

The $7^{\text {th }}$ International Workshop on $\mathrm{ZnO}$ and Related Materials, Sept. 11-14, 2012, Nice, France.

3. Magneto-optical study of donor bound exciton comprising $B$ valence band hole.

S. L. Chen, W. M. Chen, and I. A. Buyanova,

The $7^{\text {th }}$ International Workshop on $\mathrm{ZnO}$ and Related Materials, Sept. 11-14, 2012, Nice, France.

4. Optical studies and defect properties of GaP/GaNP core/shell nanowires. A. Dobrovolsky, S. L. Chen, J. Stehr, Y. J. Kuang, S. Sukrittanon, H. Li, C. W. Tu, W. M. Chen, and I. A. Buyanova,

The $13^{\text {th }}$ edition of Trends in Nanotechnology International Conference, Sept. 10-14, 2012, Madrid, Spain.

5. Realization of slow light in $\mathrm{ZnO}$ media.

S. L. Chen, W. M. Chen and I. A. Buyanova,

The $31^{\text {st }}$ International Conference on the Physics of Semiconductors, July 29Aug. 3, 2012, Zurich, Switzerland.

6. Cathodoluminescence studies of $\mathrm{ZnO}$ tetrapod structures.

S. L. Chen, S. K. Lee, W. M. Chen and I. A. Buyanova, The $2^{\text {nd }}$ Nano Today Conference, Dec.11-15, 2011, Hawaii, USA.

7. Long delays of light in $\mathrm{ZnO}$ caused by exciton-polariton propagation. S. L. Chen, W. M. Chen and I. A. Buyanova, Int. Conf. on Fundamental Optical Processes in Semiconductors, Aug.1-5, 2011, Lake Junaluska, USA.

8. Long lifetime of free excitons in $\mathrm{ZnO}$ tetrapod structures.

S. K. Lee, S. L. Chen , H. Dong, Z. Chen, W. M. Chen, and I. A. Buyanova, MRS Spring Meeting, April 5-9, 2010, San Francisco, USA. 
9. Optical Characterizations of $\mathrm{ZnO}$ Tetrapod Nanostructures. S. K. Lee, S. L. Chen , W. M. Chen, H. Dong, Z. Chen and I. A. Buyanova, The $33^{\text {rd }}$ Workshop on Compound Semiconductor Devices and Integrated Circuits, May 17-20, 2009, Malaga, Spain. 


\section{Acknowledgement}

I would like to express my sincere gratitude to two of my supervisors, Prof. Irina Buyanova and Prof. Weimin Chen, who have offered me the precious opportunity to pursue my $\mathrm{PhD}$ study in the Division of Functional Electronic Materials at Linköping University. A great deal of time and effort has been devoted to supervising me during the past 5 years by Prof. Irina Buyanova and Prof. Weimin Chen, which has shaped me from a knowledge-learning student to a knowledge-creating researcher. I have really enjoyed and benefited from the discussions on my research work together during coffee time, which are always exciting and fruitful.

I'm grateful to my lab teacher, Dr. Sunkyun Lee. It is you who with great patience and pedagogic methods taught me about photoluminescence, especially time-resolved photoluminescence spectroscopies that I had no experience with before. The skills of operating pulsed lasers that you have imparted to me help me greatly during my experimental work.

Thanks to my peer colleague Yuttapoom Puttisong. As PhD students, we often talk about scientific knowledge and ideas on research work which broaden my vision.

I owe thanks to Dr. Daniel Dagnelund, Dr. Jan Stehr, Dr. Alexandr Dobrovolsky, Dr. Joseph Cullen, Stanislav Filippov, Yuqing Huang, together with former group members Dr. Jan Beyer, Dr. Xingjun Wang, Dr. Qinjun Ren and Dr. Deyong Wang. Thank all of you very much for helping me in the research work and in creating a vibrant working atmosphere that I really cherish.

Special acknowledgement goes to Dr. Daniel Dagnelund for the translation of Populärvetenskaplig sammanfattning. Without your help, the thesis is an incomplete one.

I want as well to thank Assoc. Prof. Ivan Ivanov, who not only helped me with PL measurements and UV laser systems, but also participated in my Licentiate defense as an opponent. 
Finally and foremost, I want to express my deepest appreciation to my parents, who are always encouraging whenever I encountered difficulties and forever supportive to the decisions I've made. Thank you very much!

\author{
Shula Chen \\ Linköping University \\ October, 2013
}




\section{Contents}

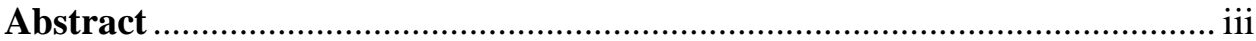

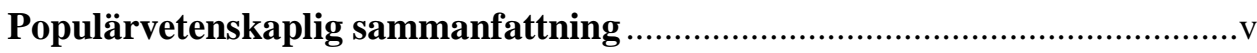

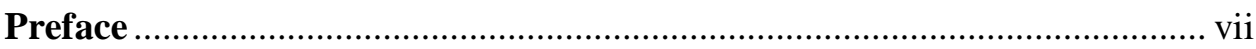

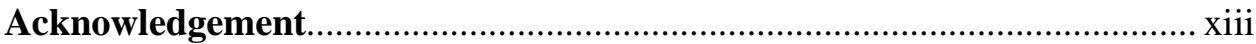

Part I. A general introduction to the research field .......................................

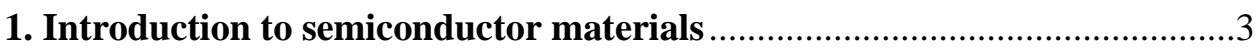

1.1 The concept of a semiconductor .................................................................

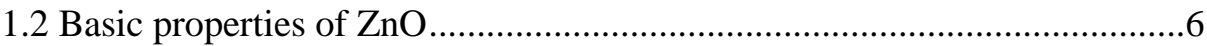

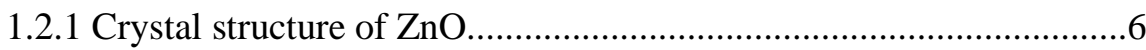

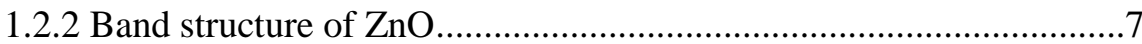

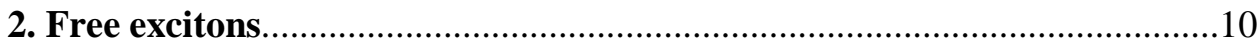

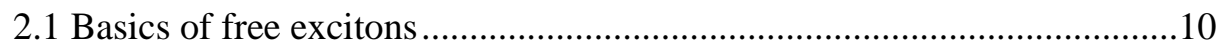

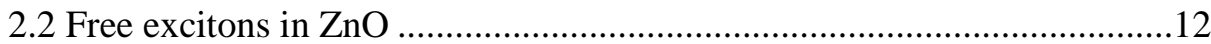

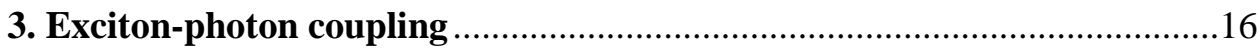

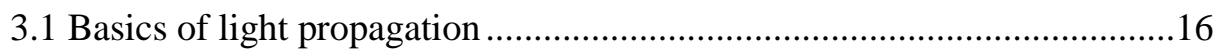

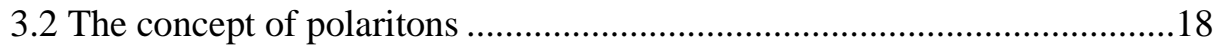

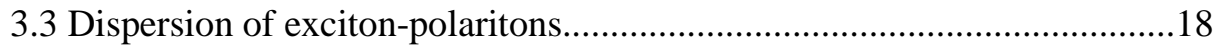

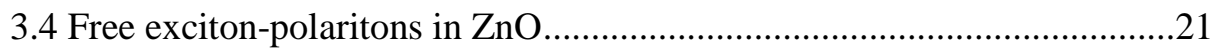

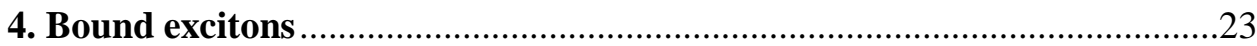

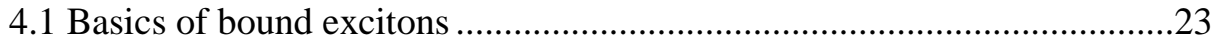

4.2 Ground and excited states of donor bound excitons in $\mathrm{ZnO}$......................25

4.3 Optical selection rules for donor bound excitons .....................................27

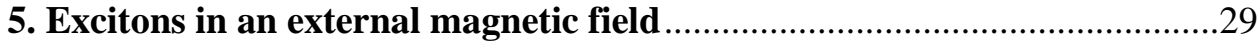

5.1 Zeeman splitting of neutral donor and acceptor bound excitons ................29

5.2 Zeeman splitting of excitons bound to isoelectronic centers and ionized

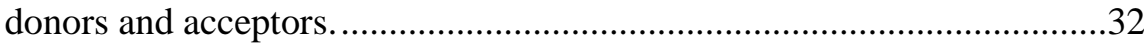




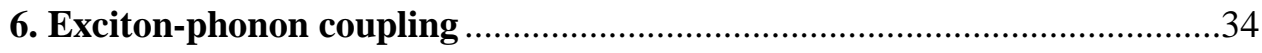

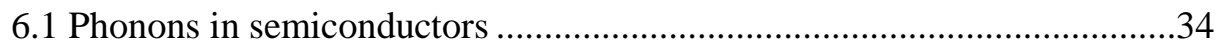

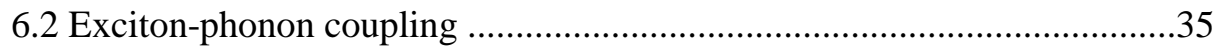

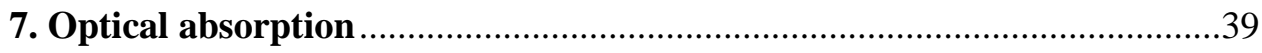

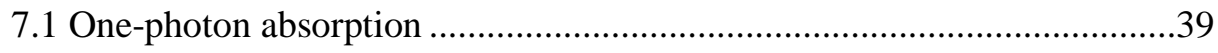

7.2 Two-photon absorption...........................................................................4

7.2.1 Absorption via a real intermediate state...........................................40

7.2.2 Absorption involving a virtual intermediate state .............................41

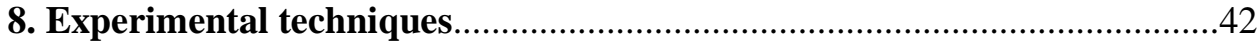

8.1 Photoluminescence spectroscopy ............................................................

8.2 Time-resolved photoluminescence spectroscopy ......................................43

8.3 Time-resolved time-of-flight technique.....................................................4

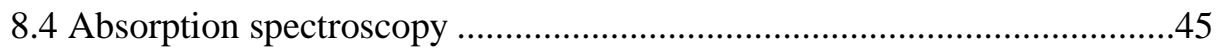

8.5 Photoluminescence excitation spectroscopy ..............................................46

8.6 Cathodoluminescence spectroscopy …………........................................4

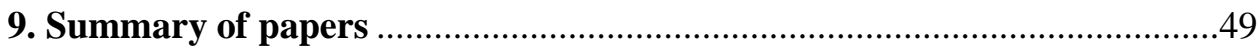

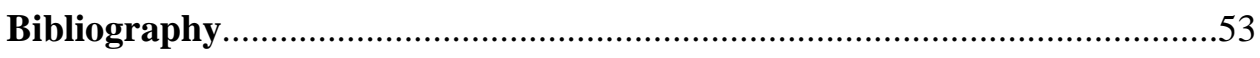

Part II. Papers...................................................... 59 


\section{Part I}

\section{A general introduction to the research field}




\section{Introduction to semiconductor materials}

\subsection{The concept of a semiconductor}

In nature, each individual atom has a discrete set of energy levels. When atoms are brought together and arranged in a periodic way to form a so-called crystal, the original discrete energy levels begin to split due to inter-atomic interactions and eventually form continuous energy bands.

The topmost band that is occupied by electrons at the absolute zero temperature $(0 \mathrm{~K})$ is commonly referred to as a valence band (VB). In semiconductors and

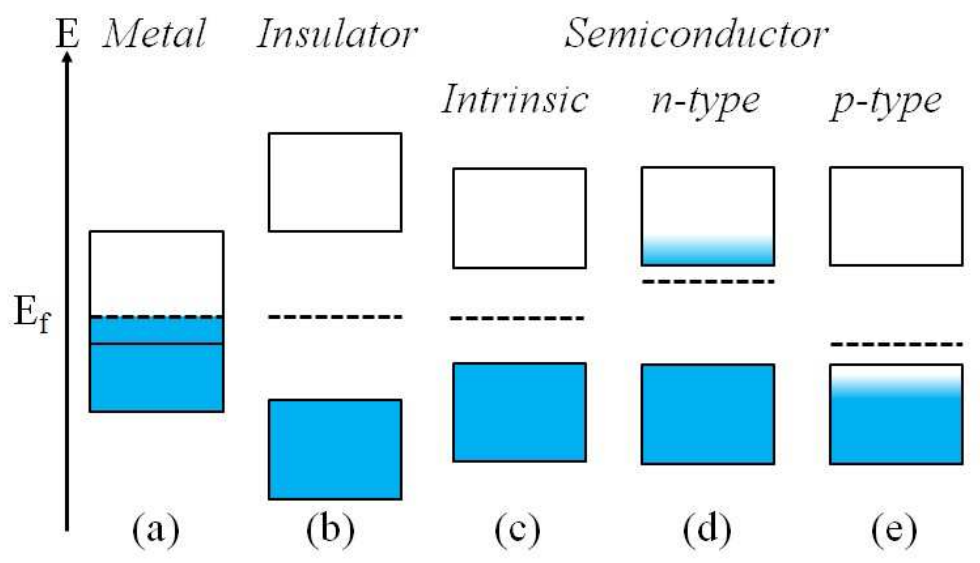

Figure 1.1 The energy band structures of (a) a metal, (b) an insulator, (c) an intrinsic semiconductor, (d) an n-type semiconductor and (e) a p-type semiconductor. The dashed lines denote Fermi levels.

insulators, this band is fully occupied and is separated from the next empty band (called conduction band (CB)) by an energy gap or a bandgap. The bandgap is a very important parameter of crystalline materials as it determines their electrical, optical and magnetic properties. When VB is partially occupied by electrons or overlaps with $\mathrm{CB}$, a crystal turns into a metal, as shown in Figure 1.1(a). Since the upmost energy band is now not fully occupied, electrons can easily move into empty energy states and, therefore, can contribute to electrical conductivity under an applied electric field. On the other hand, an insulator is obtained when the 
highest VB is full of electrons and separated from $\mathrm{CB}$ by a large bandgap, see Figure 1.1(b). Since all VB electronic states in an insulator are occupied, no charge current can be created under an electric field. However, if the bandgap of a pure insulator is comparable with thermal energy, an electron can be thermally excited from VB to the empty CB leaving an empty space in VB. This empty space is referred to as a hole and carries a positive charge. Both holes in VB and electrons in $\mathrm{CB}$ can freely move within the respective bands and contribute to electrical conductivity. Insulator materials with relatively small bandgap energies (i.e. below $\sim 4 \mathrm{eV}$ ) are called semiconductors.

A perfect semiconductor crystal without any impurities or lattice defects is called an intrinsic semiconductor. Such material will still remain a poor conductor at room temperature since it will contain only low concentrations of free electrons and holes, as shown in Figure 1.1(c). Conductivity of a semiconductor can be significantly modified by purposely introducing impurities into the crystal, or in other words, via doping. Figure 1.1(d) and (e) show an n-type and a p-type semiconductor doped with electron and hole contributing impurities, which are commonly referred to as donors and acceptors, respectively. The energy level of a donor (acceptor) typically lies in the proximity of the CB (VB) edge. It, therefore, can easily supply an electron (hole) to the respective energy band at elevated temperatures, resulting in a dramatic change of free carrier concentrations. With the advantage of easy adjustment of electrical properties,

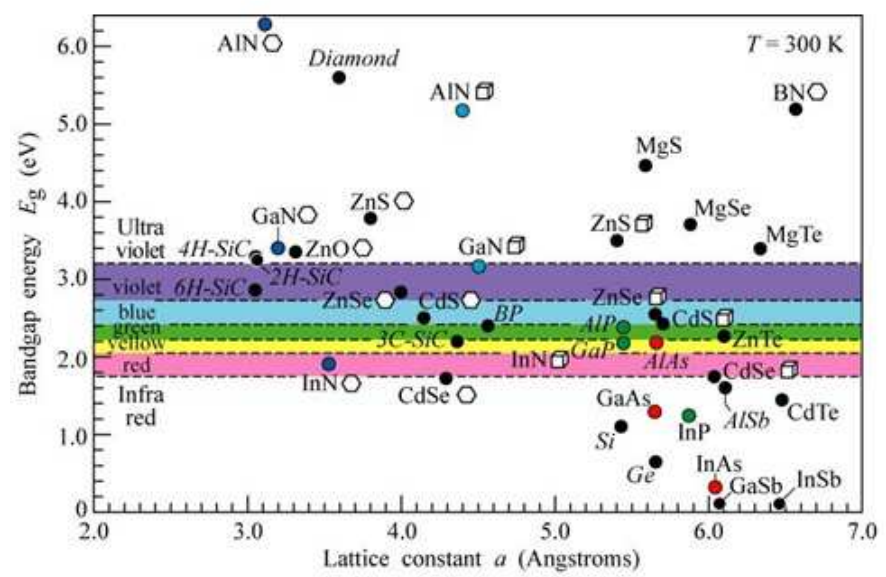

Figure 1.2 Room-temperature bandgap energies of common binary compound semiconductors vs. their lattice constants. 
semiconductors are nowadays the key materials used in the fabrication of a wide variety of electronic devices and related integrated circuits.

In addition to the prominent electrical performance, semiconductors also exhibit fascinating optical properties that are determined by their electronic structure. When a $\mathrm{CB}$ electron radiatively recombines with a VB hole in a direct gap semiconductor, a photon is emitted with an energy equal to the bandgap. Shown in Figure 1.2 [1] is a summary of some common binary compound semiconductors with different bandgap energies. One can see that by choosing different semiconductor materials or by alloying these binary compounds, the band-to-band emission could in principle be tuned within a wide spectral range from infrared to ultraviolet. Some semiconductor materials have already been successfully used to generate light in light-emitting-diodes (LED) and laser diodes (LD). On the other hand, an alternative process of light absorption can be used for light harvesting in photovoltaic solar cells, or for light detection in charge-coupled-devices (CCD) and photodiodes.

In recent years, the demand on blue to UV light emitting devices has constantly been rising driven by various applications in photonics, information storage, biology and medical therapeutics. The current commercialized solution to these needs is based on GaN. Although still hindered by problems in achieving p-type doping, $\mathrm{ZnO}$ has several advantages over $\mathrm{GaN}$ as it can be grown in large single crystals, it is amendable to wet chemical etching and it has low environmental impact and toxicity. $\mathrm{ZnO}$ is also an excellent $\mathrm{UV}$ light emitter owing to a large free exciton (FX) binding energy of $60 \mathrm{meV}$, which secures very efficient radiative $\mathrm{FX}$ recombination even at room temperature. The optical properties can be further tailored by taking advantages of bandgap engineering as $\mathrm{ZnO}$ can be synthesized with superior optical quality in various nanoscale forms ranging from two-dimensional to zero-dimensional structures [2-7]. Therefore $\mathrm{ZnO}$ can be used in emerging nano-optoelectronic devices (e.g. in UV nano-lasers) [8,9] to be implemented e.g. in novel photonic circuits.

It is under this background this thesis analyzes excitonic properties of $\mathrm{ZnO}$ which were not fully understood before and may help deepen our understanding of this material. 


\subsection{Basic properties of $\mathrm{ZnO}$}

\subsubsection{Crystal structure of $\mathrm{ZnO}$}

Crystalline materials are distinguished by the fact that all atoms that form these materials are arranged in a three-dimensional periodic fashion. A crystal structure could be constructed by attaching a basis of constituent atoms to discrete points of a periodic array (or a crystal lattice). $\mathrm{ZnO}$ crystalizes in a wurtzite structure with two lattice parameters $\mathrm{c}$ and a characterizing the unit cell, which is shown in

(a)

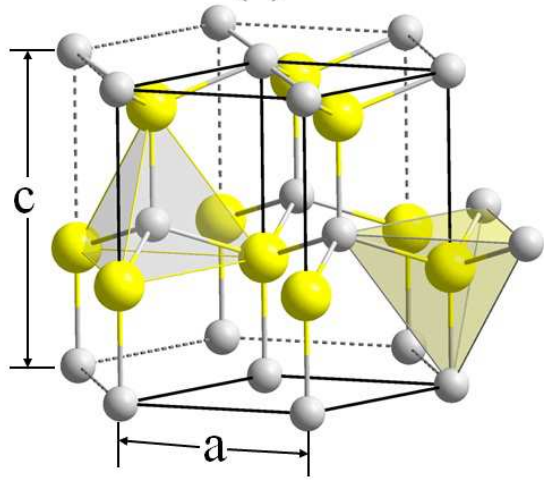

(b)

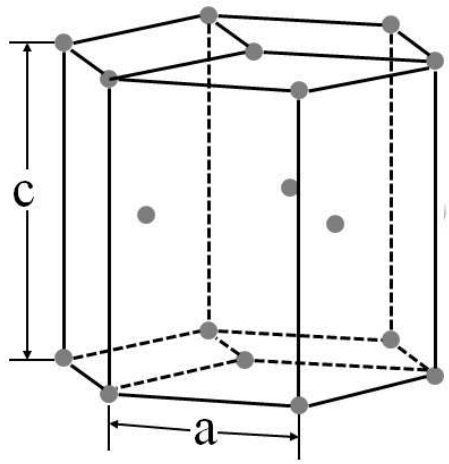

Figure 1.3 (a) Wurtzite crystal structure of $\mathrm{ZnO}(\mathrm{b})$ hexagonal-close-pack (hcp) lattice structure.

Figure 1.3(a) [10]. This structure is formed by displacing two hexagonal-closepack (hcp) $\mathrm{Zn}$ and $\mathrm{O}$ sublattices, as shown in Figure 1.3(b), along the crystallographic c-axis. Each atom in the wurtzite structure is tetrahedrally bonded to 4 neighboring atoms of the other type. With such type of crystal structure, $\mathrm{ZnO}$ possesses a symmetry that belongs to the space group of $C_{6 v}^{4}$ and is a uniaxial material which has profound effects on its electronic structure. 


\subsubsection{Band structure of $\mathrm{ZnO}$}

The conduction band of $\mathrm{ZnO}$ evolves from $4 \mathrm{~s}$ states of $\mathrm{Zn}$ atoms and has an slike Bloch wavefunction. On the other hand, the valence band is constructed from $2 p$ states of $\mathrm{O}$ atoms with a p-like Bloch wavefunction. A full description of the valence band should include both a crystal-field effect and spin-orbit interaction. The valence band Hamiltonian at the Brillouin zone center without an external magnetic field for wurtzite semiconductors could be derived from Ref. $[11,12]$ as:

$$
H_{v}=\Delta_{c f}\left(\hat{L}_{z}^{2}-1\right)+\frac{1}{3} \Delta_{s o}^{\|}\left[\left(\hat{L}_{z} \hat{\sigma}_{z}\right)-1\right]+\frac{1}{3} \Delta_{s o}^{\perp}\left(\hat{L}_{\perp} \hat{\sigma}_{\perp}\right)
$$

Here $\Delta_{c f}$ characterizes the crystal-field effect, $\Delta_{s o}^{\|}$and $\Delta_{s o}^{\perp}$ account for the anisotropic spin-orbit interaction. $\hat{L}$ and $\hat{\sigma}$ are the orbital angular momentum operator and Pauli spin matrices, respectively.

We start the discussion of the valence band evolution from the simplest case of zinc-blende structure and excluding spin-orbit interaction, as shown in Figure 1.4(a). In this case, the conduction band has $\Gamma_{1}$ symmetry with the orbital state $|s\rangle$ and the valence band has $\Gamma_{4}$ symmetry and is three-fold degenerate with

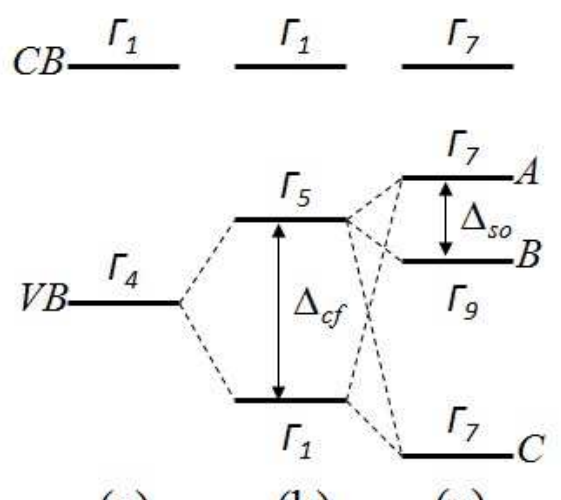

(a)

(b)

(c)

Figure 1.4 Energy level diagram of the conduction and valence band of $\mathrm{ZnO}$. (a) Valence band without crystal-field and spin-orbit interaction. (b) Valence band splitting due to a crystal-field effect. (c) Valence band splitting including both crystal-field and spin-orbit interaction. 
$|x\rangle,|y\rangle$ and $|z\rangle$ orbital states. Then, we take into consideration effects of the crystal-field on the valence band splitting. The Hamiltonian operator of the crystal-field is given as:

$$
H_{c f}=\Delta_{c f}\left(\hat{L}_{z}^{2}-1\right)
$$

From equation 1.2, it is clear that the crystal-field will lift the degeneracy between $|z\rangle$ and $|x\rangle,|y\rangle$ orbital states. Therefore, the valence band splits into two subbands as illustrated in Figure 1.4(b). The upper subband of $\Gamma_{5}$ symmetry is two-fold degenerate with the $|x\rangle,|y\rangle$ character. The lower subband is a singlet $|z\rangle$ state of the $\Gamma_{1}$ symmetry.

To complete the description of valence band, we further include the spin-orbit interaction, which reads:

$$
H_{s o}=\frac{1}{3} \Delta_{s o}^{\|}\left[\left(\hat{L}_{z} \hat{\sigma}_{z}\right)-1\right]+\frac{1}{3} \Delta_{s o}^{\perp}\left(\hat{L}_{\perp} \hat{\sigma}_{\perp}\right)
$$

and use a new set of basis functions which are the product of the orbital and spin parts as shown in Table 1.1.

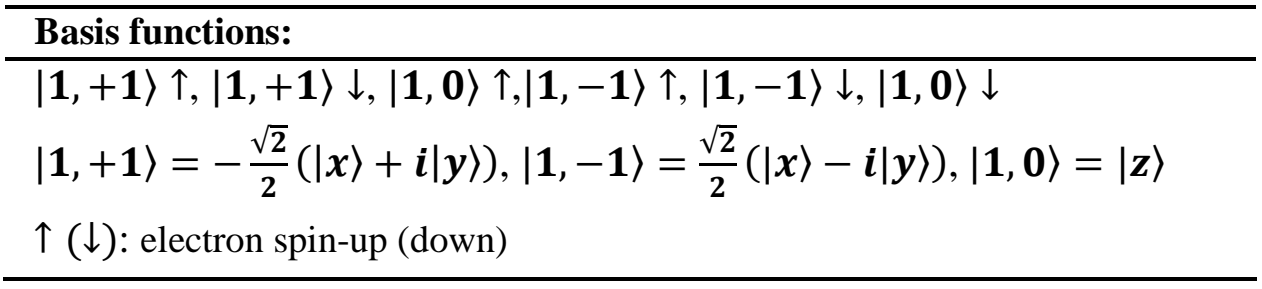

Table 1.1 Basis functions for constructing matrix representations of $H_{v}$

By solving the complete valence band Hamiltonian $H_{v}$, the eigenenergies and eigenfunctions of three spin degenerate valence subbands [11] can be derived as summarized in Table1.2. Illustrated in Figure 1.4(c) is the splitting of the valence band into $\mathrm{A}, \mathrm{B}$ and $\mathrm{C}$ subbands due to combined effects of the crystal-field and spin-orbit interactions.

The ordering of $\mathrm{A}, \mathrm{B}$ and $\mathrm{C}$ valence subbands, i.e. $\Gamma_{7}-\Gamma_{9}-\Gamma_{7}$ vs. $\Gamma_{9}-\Gamma_{7}-\Gamma_{7}$, was long-debated in the literature [11-21]. Recent experimental and theoretical studies $[11,20,21]$ have favored the $\Gamma_{7}$ symmetry of the topmost A subband, which is different from other wurtzite semiconductors like CdS and GaN. This reversed symmetry between the A and B subbands was explained as being due to 
an effective negative spin-orbit interaction induced by the $\mathrm{Zn}$ (3d) band [11]. The work presented in this thesis also adopts the $\Gamma_{7}-\Gamma_{9}-\Gamma_{7}$ ordering for the A, B, and $\mathrm{C}$ valence subbands.

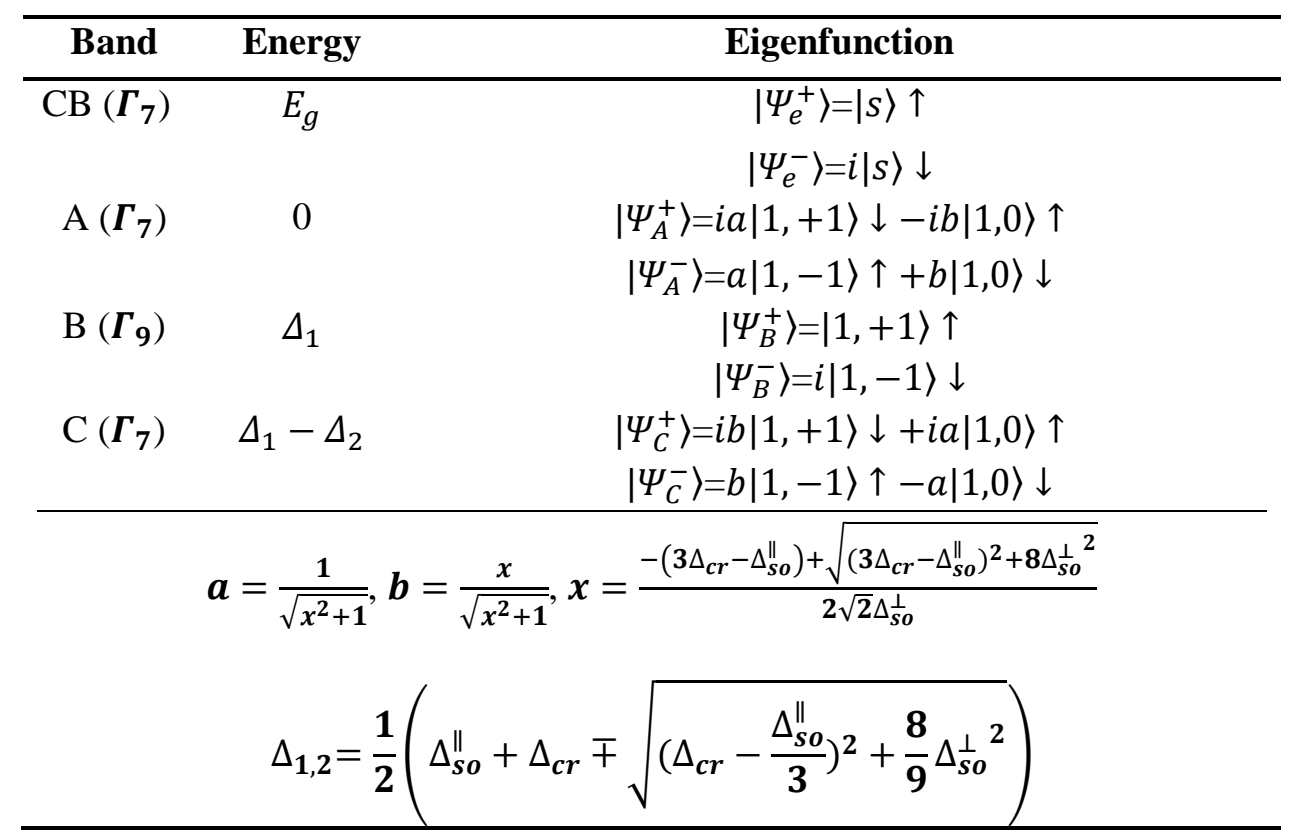

Table 1.2 Eigenstates and energies of the conduction and valence bands in $\mathrm{ZnO}$. 


\section{Free excitons}

\subsection{Basics of free excitons}

At $0 \mathrm{~K}$, an intrinsic semiconductor rests at its ground state with the valence band fully filled by electrons and the conduction band being completely empty. Upon excitation due to e.g. photon absorption, an electron can be promoted to the conduction band leaving behind a hole in the valence band. Both electron and hole can freely move in the crystal and are therefore called free carriers. Since these two types of particles possess opposite charges, the Coulomb interaction between the electron and hole is able to bind them together to form a pair which could be viewed as a quasi-particle called a free exciton (FX).

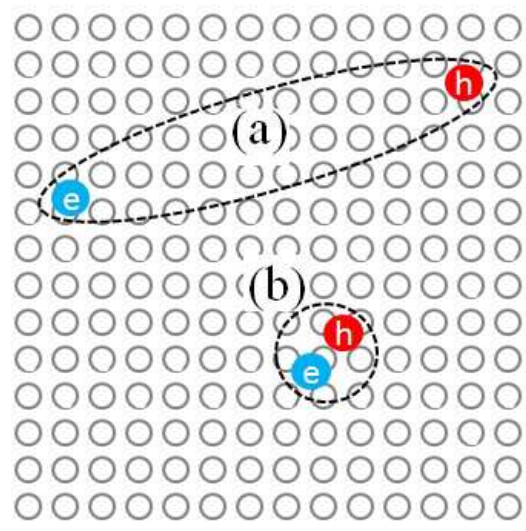

Figure 2.1 Schematic pictures of (a) Wannier-Mott exciton and (b) Frenkel exciton.

In common semiconductors with rather large dielectric constants, screening of electric field tends to weaken the Coulomb force between the electron and hole in the FX complex. As a consequence, the electron and hole are loosely bound to each other at a distance that is much larger than the unit cell. The FX of this type is called the Wannier-Mott exciton as shown in Figure 2.1(a). Since the exciton wave function in this case is spatially spread in the lattice, one can adopt an effective-mass-approximation (EMA) to explain its properties. However, strongly ionic materials like $\mathrm{NaCl}$ and $\mathrm{KI}$ have relatively small dielectric 
constants. In such materials, the binding of the electron-hole-pair is stronger than for the Wannier-Mott exciton and the spatial extension of the FX wavefunction is comparable with the size of the unit cell. This kind of FX is called the Frenkel exciton, see Figure 2.1(b). In the following, we restrict our discussion to the Wannier-Mott exciton.

Within the framework of EMA, FX could be equivalently considered as a free hydrogen atom moving in the semiconductor. As illustrated in Figure 2.2(a), its motion could be separated into a center-of-mass part due to translational movement and a hydrogen-like part describing the relative motion of the electron and hole. This model provides the energy (E)-momentum (k) dispersion as:

$$
\begin{gathered}
E_{F X}(n, k)=E_{g}-\frac{R y^{*}}{n^{2}}+\frac{\hbar^{2} k^{2}}{2 M}, \quad R_{y}{ }^{*}=\frac{-13.6(\mathrm{eV})}{\varepsilon_{r}^{2}} \frac{\mu}{m_{0}} \\
M=m_{e}+m_{h}, \mu=\frac{m_{e} m_{h}}{m_{e}+m_{h}}, k=k_{e}+k_{h}
\end{gathered}
$$

The dispersion based on the above expressions is schematically shown in Figure 2.2(b). We can see that the principal quantum number $n$ and the effective Rydberg energy $R_{y}{ }^{*}$ determine a hydrogen-like series of FX states in the gap. $R_{y}{ }^{*}$ is modified by the relative dielectric constant $\varepsilon_{r}$ and the reduced FX effective mass $\mu$. The parabolic dispersion of the kinetic energy is governed by the translational mass $M$ and the wave-vector of the exciton $k$.

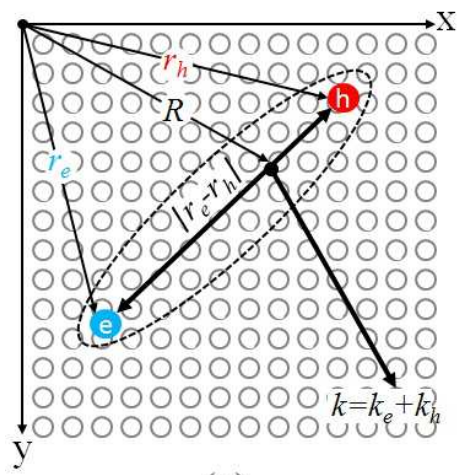

(a)

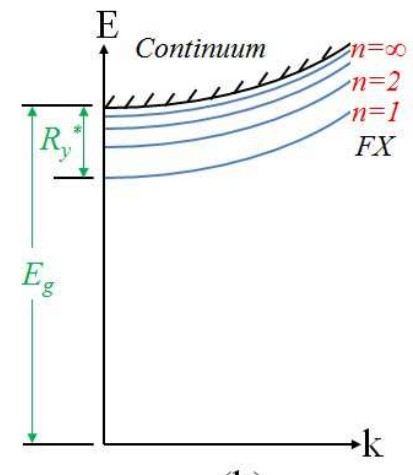

(b)

Figure 2.2 (a) Separation of FX motion into the translational part characterized by $R$ and the relative part represented by $\left|r_{e}-r_{h}\right|$. (b) FX energy levels. 
When FX is excited into a higher excited state with a larger principal quantum number, its electron and hole envelope wavefunctions become increasingly spreading in space. When FX finally reaches the excited states that are in close proximity of the continuum, the electron and hole in the FX complex dissociate and move again like uncorrelated free carriers. By measuring the energy difference between the FX ground state $(n=1)$ and the bandgap, we can obtain the FX binding energy, which is a measure of its thermal stability at elevated temperatures.

\subsection{Free excitons in $\mathrm{ZnO}$}

Since valence band of $\mathrm{ZnO}$ is split into $\mathrm{A}\left(\Gamma_{7}\right), \mathrm{B}\left(\Gamma_{9}\right)$ and $\mathrm{C}\left(\Gamma_{7}\right)$ subbands, different exciton states are formed depending on the nature of the hole state. From group theory arguments, symmetries of the corresponding states can be determined as a direct product of group representations of the related band symmetries as:

$$
\begin{gathered}
\Gamma_{7} \times \Gamma_{7} \rightarrow \Gamma_{5}(\perp)+\Gamma_{1}(\|)+\Gamma_{2} \text { for } \mathrm{FX}_{\mathrm{A}, \mathrm{C}} \\
\Gamma_{7} \times \Gamma_{9} \rightarrow \Gamma_{5}(\perp)+\Gamma_{6} \text { for } \mathrm{FX}_{\mathrm{B}}
\end{gathered}
$$

At the center of the Brillouin zone, the $\Gamma_{5}$ (doublet) and $\Gamma_{1}$ (singlet) excitons are dipole-allowed with emission polarization perpendicular and parallel to the crystallographic c-axis, respectively. The $\Gamma_{6}$ (doublet) and $\Gamma_{2}$ (singlet) transitions are dipole-forbidden and cannot be detected optically without an external perturbation like an applied magnetic field, a strain field, etc.

Quantitative interpretation of FX can be obtained by solving the following exciton Hamiltonian [22-24]:

$$
H_{\text {exc }}=H_{c}-H_{v}-R^{*}+\frac{J}{2}\left(1-\sigma_{e} \cdot \sigma_{h}\right)
$$

Here $H_{c}$ and $H_{v}$ denote conduction and valence band Hamiltonians, $R^{*}$ represents the Coulomb interaction which defines the exciton binding energy, the last term accounts for the exchange interaction where $J$ is the exchange 
interaction constant, $\sigma_{e}$ and $\sigma_{h}$ are the Pauli spin matrices of an electron and hole, respectively. The 12 exciton basis functions are defined below as:

\begin{tabular}{l}
\hline Basis functions: \\
\hline$|\mathbf{1},+\mathbf{1}\rangle \Uparrow \uparrow,|\mathbf{1},-\mathbf{1}\rangle \Uparrow \uparrow,|\mathbf{1}, \mathbf{0}\rangle \Downarrow \uparrow,|\mathbf{1},+\mathbf{1}\rangle \Downarrow \downarrow,|\mathbf{1},-\mathbf{1}\rangle \Downarrow \downarrow,|\mathbf{1}, \mathbf{0}\rangle \Uparrow \downarrow$ \\
$|\mathbf{1},+\mathbf{1}\rangle \Uparrow \downarrow,|\mathbf{1},-\mathbf{1}\rangle \Uparrow \downarrow,|\mathbf{1}, \mathbf{0}\rangle \Downarrow \downarrow,|\mathbf{1},+\mathbf{1}\rangle \Downarrow \uparrow,|\mathbf{1},-\mathbf{1}\rangle \Downarrow \uparrow,|\mathbf{1}, \mathbf{0}\rangle \Uparrow \uparrow$ \\
$\Uparrow \Downarrow(\uparrow \downarrow)$ : hole (electron) spin
\end{tabular}

Table 2.1 Basis functions for constructing matrix representations of $H_{\text {exc }}$

One can solve the exciton Hamiltonian $H_{\text {exc }}$ and derive the excitonic eigenstates [25] in $\mathrm{ZnO}$. The corresponding results at the center of the Brillouin zone are summarized in Table 2.2.

\begin{tabular}{|c|c|c|}
\hline Exciton & $\begin{array}{lll}\text { Eigenfunction } & \text { Sym } \\
\end{array}$ & netry \\
\hline \multirow{4}{*}{$\mathrm{FX}_{\mathrm{A}}$} & $\left|x_{A}\right\rangle=\frac{\sqrt{2}}{2} a(|1,+1\rangle \Downarrow \uparrow-|1,-1\rangle \Uparrow \downarrow)-\frac{\sqrt{2}}{2} b(|1,0\rangle \Uparrow \uparrow-|1,0\rangle \Downarrow \downarrow)$ & $\left(\Gamma_{5}\right)$ \\
\hline & $\left|y_{A}\right\rangle=-\frac{\sqrt{2}}{2} i a(|1,+1\rangle \Downarrow \uparrow+|1,-1\rangle \Uparrow \downarrow)+\frac{\sqrt{2}}{2} i b(|1,0\rangle \Uparrow \uparrow+|1,0\rangle \Downarrow \downarrow)$ & $\left(\Gamma_{5}\right)$ \\
\hline & $\left|z_{A}\right\rangle=-\frac{\sqrt{2}}{2} a(|1,-1\rangle \Uparrow \uparrow+|1,+1\rangle \Downarrow \downarrow)+\frac{\sqrt{2}}{2} b(|1,0\rangle \Downarrow \uparrow+|1,0\rangle \Uparrow \downarrow)$ & $\left(\Gamma_{1}\right)$ \\
\hline & $\left|t_{A}\right\rangle=\frac{\sqrt{2}}{2} i a(|1,-1\rangle \Uparrow \uparrow-|1,+1\rangle \Downarrow \downarrow)-\frac{\sqrt{2}}{2} i b(|1,0\rangle \Downarrow \uparrow-|1,0\rangle \Uparrow \downarrow)$ & $\left(\Gamma_{2}\right)$ \\
\hline \multirow{4}{*}{$\mathrm{FX}_{\mathrm{B}}$} & $\left|x_{B}\right\rangle=\frac{\sqrt{2}}{2}(|1,-1\rangle \Downarrow \uparrow-|1,+1\rangle \Uparrow \downarrow)$ & $\left(\Gamma_{5}\right)$ \\
\hline & $\left|y_{B}\right\rangle=\frac{\sqrt{2}}{2} i(|1,-1\rangle \Downarrow \uparrow+|1,+1\rangle \Uparrow \downarrow)$ & $\left(\Gamma_{5}\right)$ \\
\hline & $\left|t_{B}\right\rangle=\frac{\sqrt{2}}{2} i(|1,+1\rangle \Uparrow \uparrow-|1,-1\rangle \Downarrow \downarrow)$ & $\left(\Gamma_{6}\right)$ \\
\hline & $\left|t_{B}^{\prime}\right\rangle=\frac{\sqrt{2}}{2}(|1,+1\rangle \Uparrow \uparrow+|1,-1\rangle \Downarrow \downarrow)$ & $\left(\Gamma_{6}\right)$ \\
\hline \multirow{4}{*}{$\mathrm{FX}_{\mathrm{C}}$} & $\left|x_{C}\right\rangle=\frac{\sqrt{2}}{2} b(|1,+1\rangle \Downarrow \uparrow-|1,-1\rangle \Uparrow \downarrow)-\frac{\sqrt{2}}{2} a(|1,0\rangle \Uparrow \uparrow-|1,0\rangle \Downarrow \downarrow)$ & $\left(\Gamma_{5}\right)$ \\
\hline & $\left|y_{C}\right\rangle=-\frac{\sqrt{2}}{2} i b(|1,+1\rangle \Downarrow \uparrow-|1,-1\rangle \Uparrow \downarrow)+\frac{\sqrt{2}}{2} i a(|1,0\rangle \Uparrow \uparrow-|1,0\rangle \Downarrow \downarrow)$ & $\left(\Gamma_{5}\right)$ \\
\hline & $\left|z_{A}\right\rangle=-\frac{\sqrt{2}}{2} b(|1,-1\rangle \Uparrow \uparrow+|1,+1\rangle \Downarrow \downarrow)+\frac{\sqrt{2}}{2} a(|1,0\rangle \Downarrow \uparrow+|1,0\rangle \Uparrow \downarrow)$ & $\left(\Gamma_{1}\right)$ \\
\hline & $\left|t_{A}\right\rangle=\frac{\sqrt{2}}{2} i b(|1,-1\rangle \Uparrow \uparrow-|1,+1\rangle \Downarrow \downarrow)-\frac{\sqrt{2}}{2} i a(|1,0\rangle \Downarrow \uparrow-|1,0\rangle \Uparrow \downarrow)$ & $\left(\Gamma_{2}\right)$ \\
\hline
\end{tabular}

Table 2.2 Eigenfunctions of $F X_{A}, F X_{B}$ and $F X_{C}$ derived from the exciton Hamiltonian $H_{\text {exc }}$. The prefactors of $a$ and $b$ are the same as those used in the valence band eigenfunctions. 
From the FX eigenfunctions, one can easily determine dipole polarization and oscillator strength associated with each type of the exciton. The doubly degenerate $\Gamma_{5}$ exciton states of $\mathrm{FX}_{\mathrm{A}}$ and $\mathrm{FX}_{\mathrm{C}}$ are mixed spin singlet and triplet states with two dipole components $(|1,+1\rangle \Downarrow \uparrow \pm|1,-1\rangle \Uparrow \downarrow)$ and $(|1,0\rangle \Uparrow \uparrow$ $\pm|1,0\rangle \Downarrow \downarrow)$. The singlet component is a linear combination of orbital states $|1,+1\rangle$ and $|1,-1\rangle$, which have dipoles oriented along the $\mathrm{x}$ and $\mathrm{y}$ axis, respectively. Therefore, the transition of $\Gamma_{5}$ exciton emits light with $\mathrm{E} \perp \mathrm{c}$ (the zaxis is directed along the c-axis of crystal). The triplet component contains the orbital part $|1,0\rangle$ whose dipole emits along the c-axis. However, due to the parallel orientation of hole and electron spins, this dipole-allowed transition is spin-forbidden.

The singly degenerate $\Gamma_{1}$ exciton has similar orbital wavefunction as the $\Gamma_{5}$ exciton. But now the spin-allowed singlet component consists of the dipole component $(|1,0\rangle \Downarrow \uparrow+|1,0\rangle \Uparrow \downarrow)$ along the c-axis and the triplet component with $(|1,-1\rangle \Uparrow \uparrow+|1,+1\rangle \Downarrow \downarrow)$, which is spin-forbidden. Consequently, the $\Gamma_{1}$ exciton only emits in the E\|c polarization.

As to the $\Gamma_{2}$ and $\Gamma_{6}$ excitons, they are both spin triplet states which are optically inactive. Therefore, these states are normally dark at the center of the Brillouin zone without external perturbations like strain, magnetic field, etc.

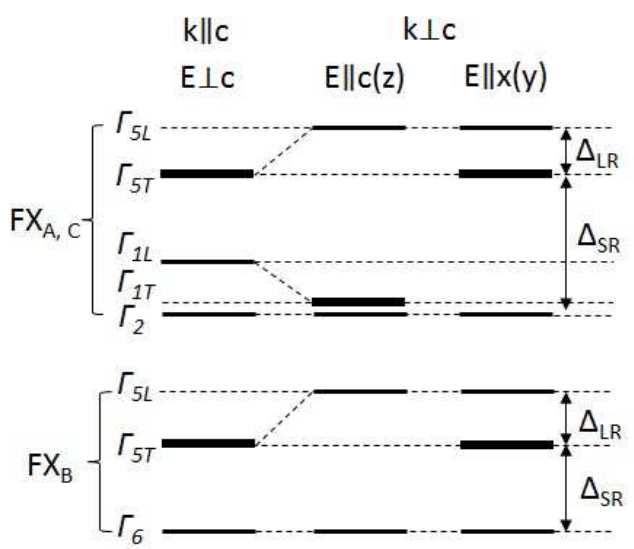

Figure 2.3 Energy level scheme of all exciton states. The solid lines denote involved excitons in the specified geometry. The thick and thin lines stand for allowed and forbidden transitions, respectively. $\Delta_{S R}$ and $\Delta_{L R}$ denote energy splitting induced by the short-range and long-range exchange interactions, respectively. 
Let us now discuss energies of different FX states. Exchange interaction between the electron and hole within the FX lifts the degeneracy between exciton states with different total spin. Exchange interaction comprises a short range (analytical) term and a long range (non-analytical) term. For $\mathrm{FX}_{\mathrm{A}}$ and $\mathrm{FX}_{\mathrm{C}}$ in $\mathrm{ZnO}$, the short range exchange interaction lifts the energy level of the $\Gamma_{5}$ exciton above the $\Gamma_{1}$ and $\Gamma_{2}$ exciton states. Similarly for $\mathrm{FX}_{\mathrm{B}}$, the $\Gamma_{5}$ exciton state is located above the $\Gamma_{6}$ exciton. By further taking into account the long range exchange interaction which is related to the translational wave-vector $\mathrm{k}$ of exciton, the dipole-allowed $\Gamma_{5}$ and $\Gamma_{1}$ excitons would undergo additional splitting into transverse and longitudinal exciton states, i.e. $\Gamma_{5 T}$ and $\Gamma_{5 L}, \Gamma_{1 T}$ and $\Gamma_{1 L}$. The energy level diagram of all FX states which takes into account the exchange interaction, wave-vector orientation and dipole polarization is illustrated in Figure 2.3. 


\section{Exciton-photon coupling}

\subsection{Basics of light propagation}

In nature, the propagation of light follows the famous Maxwell's laws:

$$
\begin{gathered}
\nabla \cdot D=\rho, \nabla \cdot B=0, \nabla \times E=-\frac{\partial B}{\partial t}, \nabla \times H=J+\frac{\partial D}{\partial t} \\
D=\varepsilon_{0} \varepsilon_{r} E, B=\mu_{0} \mu_{r} H
\end{gathered}
$$

$D$ : electric displacement, $E$ : electric field, $B$ : magnetic flux density, $H$ : magnetic field strength, $\rho$ : charge density, $J$ : current density, $\varepsilon_{0}\left(\varepsilon_{r}\right)$ : vacuum (relative) permittivity, $\mu_{0}\left(\mu_{r}\right)$ : vacuum (relative) permeability.

When light is travelling in vacuum where $\rho=0$ and $J=0$, the Maxwell equations can be re-arranged into the well-known Helmholtz equation with $E$ expressed as:

$$
\nabla^{2} E-\mu_{0} \varepsilon_{0} \frac{\partial^{2} E}{\partial t^{2}}=0
$$

One of simple solutions to equation 3.3 is the plane wave of the form:

$$
E(r, t)=E_{0} \exp [i(k \cdot r-\omega t)]
$$

where $k$ and $\omega$ are wave vector and angular frequency of the plane wave. Since there is no net charge in vacuum, according to the Gauss law,

$$
\nabla \cdot E=0
$$

By substituting equation 3.4 into equation 3.5 , we can get:

$$
\nabla \cdot E=i E_{0} \cdot k \exp [i(k \cdot r-\omega t)]=0
$$

This explicitly shows that

$$
E_{0} \cdot k=0
$$


This means that the electric field vector of the propagating light is perpendicular to its wave vector, i.e. $E_{0} \perp k$. In such case, the plane wave of light is a transverse wave with respect to $E_{0}$.

When light is propagating inside a non-conductive dielectric medium, the Helmholtz equation of the electric field component changes the form to:

$$
\begin{aligned}
& \nabla^{2} E-\mu_{0} \varepsilon_{0} \frac{\partial^{2} E}{\partial t^{2}}=\mu_{0} \frac{\partial^{2} P}{\partial t^{2}} \\
& P=\varepsilon_{0} \chi E=\varepsilon_{0}\left(\varepsilon_{r}-1\right) E
\end{aligned}
$$

This equation tells that the electric field in matter is accompanied by the polarization field $P$. By inserting equation 3.9 into equation 3.8 and re-arranging it, we get a new form which is similar to equation 3.3:

$$
\nabla^{2} E-\mu_{0} \varepsilon_{0} \varepsilon_{r}(\omega) \frac{\partial^{2} E}{\partial t^{2}}=0
$$

One of common solutions to equation 3.8 is again the plane wave with transverse electric field. In addition, there is a new class of solutions which also obey the Gauss law

$$
\nabla \cdot D=\nabla \cdot \varepsilon_{0} \varepsilon_{r}(\omega) E=0
$$

but with

$$
\varepsilon_{r}\left(\omega_{L}\right)=0
$$

Here $\omega_{L}$ is the angular frequency at which relative dielectric constant goes to zero. The solution at $\omega_{L}$ has electric field $E$ parallel to the wave vector $k$, therefore it is a longitudinal wave. As can be seen from the above derivation, the longitudinal wave does not exist in vacuum and is a polarization wave instead of the electromagnetic field. The longitudinal polarization wave produces an internal electric field along the propagation direction, which could be considered as a restoring force to the oscillating dipoles.

The above discussion gives a general introduction to light propagation in vacuum and matter. Further information regarding the propagation of light can be found in Ref. [26,27]. 


\subsection{The concept of polaritons}

In a classical picture, the electric field of incident light excites oscillations of polarization or dipoles in matter. The excited polarization (or dipole) will in turn emit light which acts back onto the incident field. This coupling leads to the admixture or superposition of light and polarization field which is called 'Polariton'. The energy quanta of polariton could be viewed as Boson-like quasiparticles. In the following part, we shall focus on the coupling between light and the polarization field induced by excitons in semiconductors, i.e. properties of exciton-polaritons.

\subsection{Dispersion of exciton-polaritons}

All elementary excitations in a semiconductor could be described by two physical quantities, i.e. wave-vector $(k)$ and Energy $\left(E_{\omega}\right)$. The connection between $E_{\omega}$ and $k$ gives the so-called dispersion relation, $E_{\omega}(k)$. As is shown in Figure 3.1(a), the dispersion of light in vacuum is just a straight line with a slope of $\hbar c$. For exciton states, let us start with a simple case of an exciton without translational motion. Its dispersion is a flat band over the $E_{\omega}-k$ coordinates. When light penetrates a semiconductor and interacts with an exciton to form an exciton-polariton, the originally independent photon and exciton states become mixed. The resulting exciton-polariton dispersion then has a dual character of both photon and exciton states, which could be derived from classical physics as follows.

When light is travelling in matter its wave-vector $\tilde{k}$ is related to the wave-vector in vacuum $k_{0}$ as

$$
\begin{gathered}
\tilde{k}=\tilde{n} k_{0}=\tilde{n} \frac{\omega}{c}=\tilde{n} \frac{E_{\omega}}{\hbar c} \\
\tilde{n}=n+i \kappa
\end{gathered}
$$

Here $\tilde{n}$ is a complex refractive index. Its real part, $n$, is usually referred to as the refractive index and determines light dispersion in matters. The imaginary part, $\kappa$, is the extinction coefficient that affects the absorption of light. $E_{\omega}$ and $c$ are the 


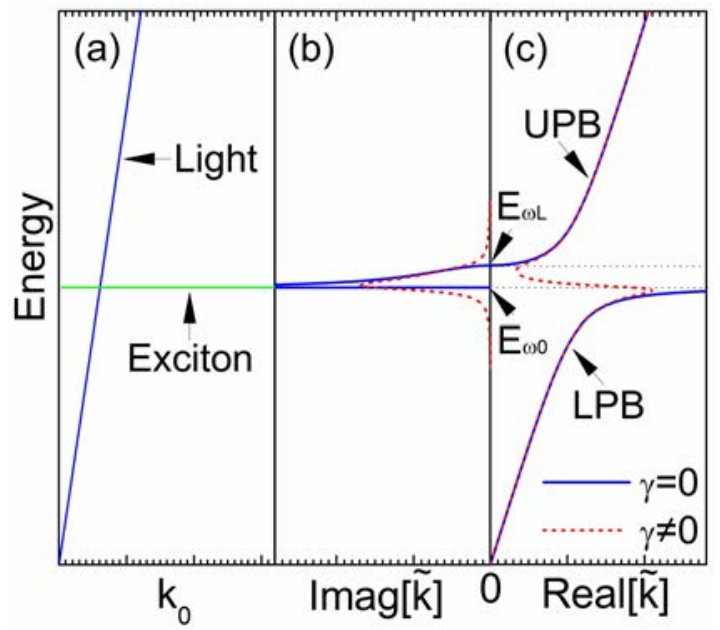

Figure 3.1 (a) Schematic dispersion relation of light in vacuum and exciton without translational motion. Imaginary and real parts of the E-k dispersion are shown in (b) and (c) respectively for exciton-polariton. The solid and dashed lines represent zero and finite damping. $E_{\omega 0}$ and $E_{\omega L}$ are marked by horizontal dotted line.

photon energy and vacuum speed of light, respectively. We can now take a further step by squaring equation 3.13 and replacing $\tilde{n}$ with a dielectric function $\varepsilon_{r}(\omega)[27,28]$, which gives:

$$
\begin{gathered}
\frac{\hbar^{2} c^{2} \tilde{k}^{2}}{E_{\omega}^{2}}=\varepsilon_{r}(\omega)=\varepsilon_{b}+\sum_{i} \frac{f_{i} E_{\omega 0 i}^{2}}{E_{\omega 0 i}^{2}-E_{\omega}^{2}-i E_{\omega} \hbar \gamma_{i}} \\
f=\varepsilon_{b}\left(\frac{E_{\omega L}^{2}}{E_{\omega 0}^{2}}-1\right)
\end{gathered}
$$

Here, $\varepsilon_{b}$ is the background dielectric constant, $f, E_{\omega 0}\left(E_{\omega L}\right)$ and $\gamma$ are the oscillator strength, transverse eigenenergy (longitudinal energy) and damping constant of the dipole oscillator in a semiconductor. The equation derived above is the so-called polariton equation which describes the $E_{\omega}-k$ relation of the polariton.

Shown in Figure 3.1(b-c) is the polariton dispersion in the vicinity of a single exciton resonance. We first look at the condition of zero exciton damping i.e. $\gamma=0$, shown by the solid line. At the lower energy side for the real part of $\tilde{k}$, the polariton dispersion keeps straight. Since it is similar to that of light in 
vacuum, this part of the dispersion is considered to be photon-like. When approaching closer to the dipole resonance at the energy $E_{\omega 0}$, the dispersion starts to become curved towards the exciton dispersion which is horizontal. This flat part is therefore called exciton-like. The whole dispersion including both photon-like and exciton-like parts constitutes the so-called lower-polaritonbranch (LPB). At the higher energy side, there is the second branch developed from the energy position $E_{\omega L}$ of the longitudinal exciton. This branch is called the upper-polariton-branch (UPB). In between $E_{\omega 0}$ and $E_{\omega L}$, there is no excitonpolariton mode. This is not surprising, since this energy range corresponds to strong absorption which is reflected by the singularity of the imaginary part of $\tilde{k}$ (see Figure 3.1(b)). If we allow for a finite value of exciton damping, the dispersion changes as shown by the dashed lines. Now the exciton-polariton mode becomes allowed between $E_{\omega 0}$ and $E_{\omega L}$, as is shown by the dashed line in Figure 3.1(c).

By examining the exciton-polariton dispersion with zero damping, we could clearly see a gap between LPB and UPB. This gap results from the coupling of

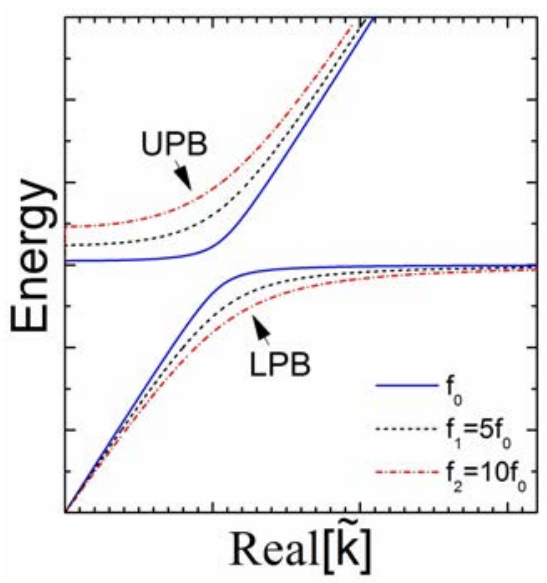

Figure 3.2 Schematic illustrating changes in the exciton-polariton dispersion with increasing oscillator strength. The exciton damping is set to zero.

the photon with exciton and therefore is strongly dependent on the coupling strength. With higher photon densities or larger exciton oscillator strength, the splitting between UPB and LPB becomes larger. This effect is illustrated in Figure 3.2. 
For FX, the exciton dispersion is a parabolic band rather than a straight horizontal level, as was already discussed in Sec.2.1. This introduces an additional correction to the polariton equation. The so-called spatial dispersion term $[26,27,29,30]$ describing exciton kinetic energy should be added to the original eigenenergy:

$$
E_{\omega 0}^{\prime}=E_{\omega 0}+\frac{\hbar^{2} k^{2}}{2 M}
$$

Then, the FX-polariton equation can be rewritten as:

$$
\frac{\hbar^{2} c^{2} \tilde{k}^{2}}{E_{\omega}^{2}}=\varepsilon_{r}(\omega)=\varepsilon_{b}+\sum_{i} \frac{f_{i} E_{\omega 0 i}^{2}}{\left(E_{\omega 0 i}+\frac{\hbar^{2} k^{2}}{2 M_{i}}\right)^{2}-E_{\omega}^{2}-i E_{\omega} \hbar \gamma_{i}}
$$

Since changes in the dielectric constant induced by the FX dispersion are quite small near the center of the Brillouin zone, the dispersion term is often neglected in the modeling of exciton-polaritons [31,32].

\subsection{Free exciton-polaritons in $\mathrm{ZnO}$}

Since the interaction strength between the photon and exciton depends on the oscillator strength of the exciton, it should also be affected by light polarization. We should recall that FXs have different dipole orientations. The $\Gamma_{5}$ excitons of $\mathrm{FX}_{\mathrm{A}}, \mathrm{FX}_{\mathrm{B}}$ and $\mathrm{FX}_{\mathrm{C}}$ are dipole-polarized perpendicular to the c-axis and, therefore, they will only couple with light polarized with $\mathrm{E} \perp \mathrm{c}$. On the other hand, the $\Gamma_{1}$ excitons of $\mathrm{FX}_{\mathrm{A}}$ and $\mathrm{FX}_{\mathrm{C}}$ are dipole-polarized along the c-axis and will be involved in the polariton formation when E\|c. Since the oscillator strength of $\mathrm{FX}_{\mathrm{C}}-\Gamma_{5}$ and $\mathrm{FX}_{\mathrm{A}}-\Gamma_{1}$ excitons is quite small, their interaction with light is fairly weak and is usually neglected in the description of the FX-polaritons. The dispersions of the FX-polariton for light polarized with $\mathrm{E} \perp \mathrm{c}$ and $\mathrm{E} \| \mathrm{c}$ are shown in Figure 3.3 by the solid and dash-dotted lines, respectively. 


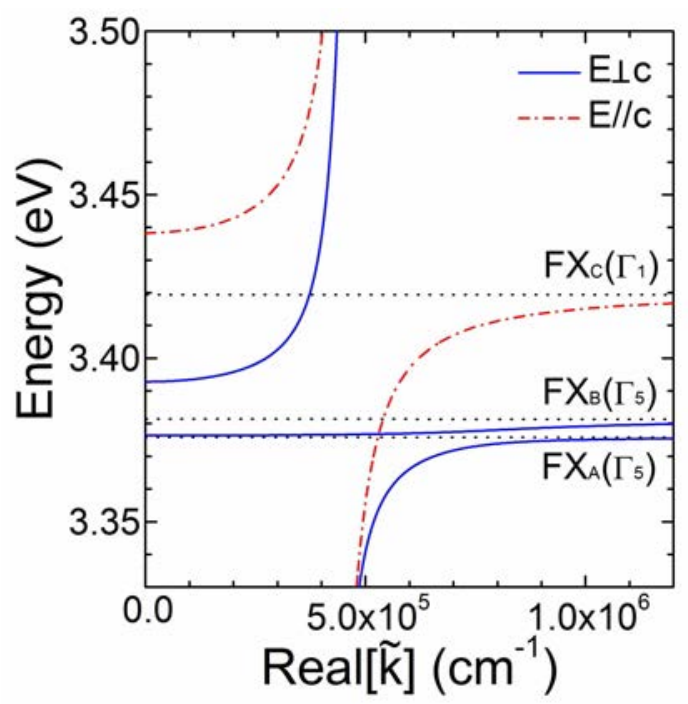

Figure 3.3 Schematic of the FX-polariton dispersion. The solid lines denote the dispersions formed by the $\Gamma_{5}$ excitons of $F X_{A}$ and $F X_{B}$ in $E \perp c$, while the dashdotted lines represent dispersions formed by the $\Gamma_{1}$ exciton of $F X_{C}$ in $E / / c$.

When the angle $\theta$ of $\mathrm{E}$ to the c-axis lies between $0^{\circ}$ and $90^{\circ}$, the transversal and longitudinal states of $\Gamma_{5}$ and $\Gamma_{1}$ excitons are coupled to form mixed-mode exciton-polaritons [33-36]. In such case, the oscillator strength of the $\Gamma_{5}$ and $\Gamma_{1}$ excitons in the mixed-mode strongly depends on $\theta$ as

$$
\begin{gathered}
f_{\Gamma_{5}}^{i}=\varepsilon_{b}\left(\frac{E_{\omega L}^{2}}{E_{\omega 0}^{2}}-1\right) \sin ^{2} \theta\left(i=F X_{A}, F X_{B} \text { and } F X_{C}\right) \\
f_{\Gamma_{1}}^{i}=\varepsilon_{b}\left(\frac{E_{\omega L}^{2}}{E_{\omega 0}^{2}}-1\right) \cos ^{2} \theta\left(i=F X_{A} \text { and } F X_{C}\right)
\end{gathered}
$$

In the end, it should be noted that when the exciton couples with light, the exciton-polariton becomes the new eigenstate to describe the interacting system. 


\section{Bound excitons}

\subsection{Basics of bound excitons}

In a perfect semiconductor, moving FXs and free carriers sense only the uniform periodic crystal potential. In the framework of the effective-mass-approximation, we could regard them as moving in the 'vacuum' space by adopting the concept of electron and hole effective mass. If there are any crystal imperfections that break periodicity of the crystal potential, defect levels would be introduced within the bandgap and create a local potential different from the host matrix. Mobile FXs and free carriers could be captured by these defect potentials and form bound excitons (BXs).

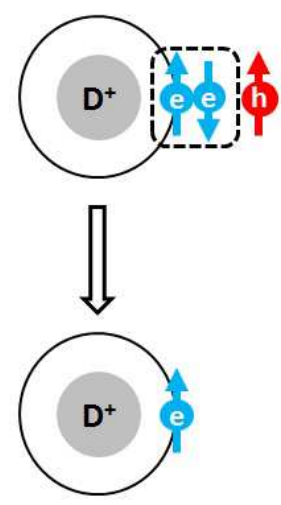

(a)

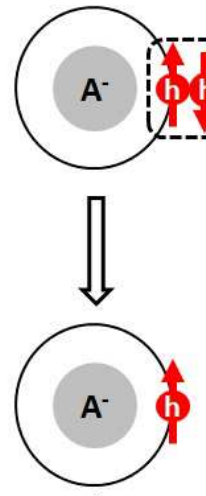

(b)
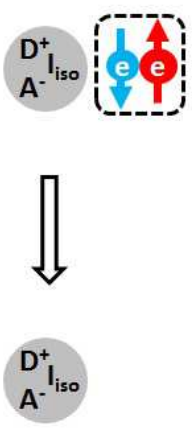

(c)

Figure 4.1 Schematic pictures of the ground and excited states of (a) $D^{0} X$, (b) $A^{0} X$ and $(c)\left[D^{+}, A^{-}, I_{i s o}\right] X$.

Different types of BX complexes could be obtained depending on the electronic structure of capturing centers. These centers are generally categorized according to the number of valence electrons as (1) isoelectronic centers which have the same number of valence electrons as substituted host atoms, (2) donors (D) and (3) acceptors (A) which have more and less valence electrons than the host atoms they are replacing, respectively. Depending on the position of the Fermi level, donors and acceptors could be either in neutral $\left(\mathrm{D}^{0}\right.$ and $\left.\mathrm{A}^{0}\right)$ or ionized $\left(\mathrm{D}^{+}\right.$and $\left.\mathrm{A}^{-}\right)$ 
charge states. In the latter case, there is no particle bound to the ionized core of the impurity atom.

Different electronic configurations of $\mathrm{BX}$ complexes at these capturing centers are shown in Figure 4.1. In the neutral donor bound exciton $\left(\mathrm{D}^{0} \mathrm{X}\right)$ complex, see Figure $4.1(\mathrm{a}), \mathrm{D}^{0}$ has a screened short range electron attractive potential which initially captures an electron and become negatively charged. The resulting long range Coulomb potential then binds a hole to neutralize the negative charge. Therefore, the resulting $\mathrm{D}^{0} \mathrm{X}$ complex consists of two electrons, which are spinpaired, and one hole. When $\mathrm{D}^{0} \mathrm{X}$ undergoes a radiative transition, the hole in the complex recombines with the electron carrying opposite spin and leaves one electron in the $1 \mathrm{~S}$ ground state after recombination. This process gives rise to the $\mathrm{D}^{0} \mathrm{X}$ emission. It is also possible that the donor electron is left in an excited state e.g. $2 \mathrm{~S}$ and $2 \mathrm{P}$ state, with the excitation energy provided by the emitted photon. This gives rise to the so-called two-electron-satellite (TES) emission, the energy of which is lower than that for the direct $\mathrm{D}^{0} \mathrm{X}$ transition. From the energy spacing $\Delta E$ between $\mathrm{D}^{0} \mathrm{X}$ and the TES (2P) transition, the donor binding energy $E_{D}$ could be derived, which is $\frac{4}{3} \Delta E$ without considering a central cell effect.

The recombination scenario of $\mathrm{D}^{0} \mathrm{X}$ holds as well for an exciton bound to neutral acceptor $\left(\mathrm{A}^{0} \mathrm{X}\right)$. In this case, however, the electron becomes spin-unpaired and one hole is left at the acceptor site after recombination as shown in Figure 4.1(b). For excitons bound to either an isoelectronic center $\left(\mathrm{I}_{\text {iso }} \mathrm{X}\right)$ or to an ionized donor and an ionized acceptor $\left(\mathrm{D}^{+} \mathrm{X}\right.$ and $\left.\mathrm{AX}\right)$, there is no particle bound at impurity atom before the BX formation. Therefore, the electronic structures of $\mathrm{I}_{\mathrm{iso}} \mathrm{X}, \mathrm{D}^{+} \mathrm{X}$ and $\mathrm{A} X$ are quite similar as shown in Figure 4.1(c).

We note that although extensive PL studies have been performed for near band edge $\mathrm{BX}$ transitions, existence of acceptor bound excitons in $\mathrm{ZnO}$ is not yet proven. Therefore the following discussion of BX properties is mainly focused on donor bound excitons.

In terms of energy positions, $\mathrm{BX}$ is separated from $\mathrm{FX}_{\mathrm{A}}$ by a localization energy $E_{l o c}$. In $\mathrm{ZnO}, \mathrm{D}^{+} \mathrm{X}$ has a higher energy level than the corresponding $\mathrm{D}^{0} \mathrm{X}$ which results in:

$$
E_{l o c}\left(\mathrm{D}^{+} \mathrm{X}\right)<E_{l o c}\left(\mathrm{D}^{0} \mathrm{X}\right)
$$


This energy sequence also holds for CdS [37]. However, depending on the effective mass ratio of electron and hole $\sigma=m_{e} / m_{h}$, the energy positions of $\mathrm{D}^{+} \mathrm{X}$ and $\mathrm{D}^{0} \mathrm{X}$ could be switched. For example in $\mathrm{ZnSe}$ [38], the trend becomes

$$
E_{l o c}\left(\mathrm{D}^{0} \mathrm{X}\right)<E_{l o c}\left(\mathrm{D}^{+} \mathrm{X}\right)
$$

which is also proposed for $\mathrm{GaN}$ [39].

\subsection{Ground and excited states of donor bound excitons in $\mathrm{ZnO}$}

The low temperature photoluminescence of $\mathrm{ZnO}$ is always dominated by a series of sharp lines within a narrow range of $30 \mathrm{meV}$ below the $\mathrm{FX}_{\mathrm{A}}$ transitions. All these emission lines originate from $\mathrm{BX}$ recombination, most of which are due to either $\mathrm{D}^{0} \mathrm{X}$ or $\mathrm{D}^{+} \mathrm{X}$. The DX PL can originate from either ground or excited exciton states. In the ground state, the hole in DX originates from the A-valence subband i.e. $\mathrm{D}^{0} \mathrm{X}^{\mathrm{A}}$ or $\mathrm{D}^{+} \mathrm{X}^{\mathrm{A}}$. The excited states could be divided into three classes [40] which are (a) rotator-vibrator states, (b) excitons that involve a B-valence band hole, i.e. $\mathrm{D}^{0} \mathrm{X}^{\mathrm{B}}$, and (c) electronic excited states. The energy levels of both

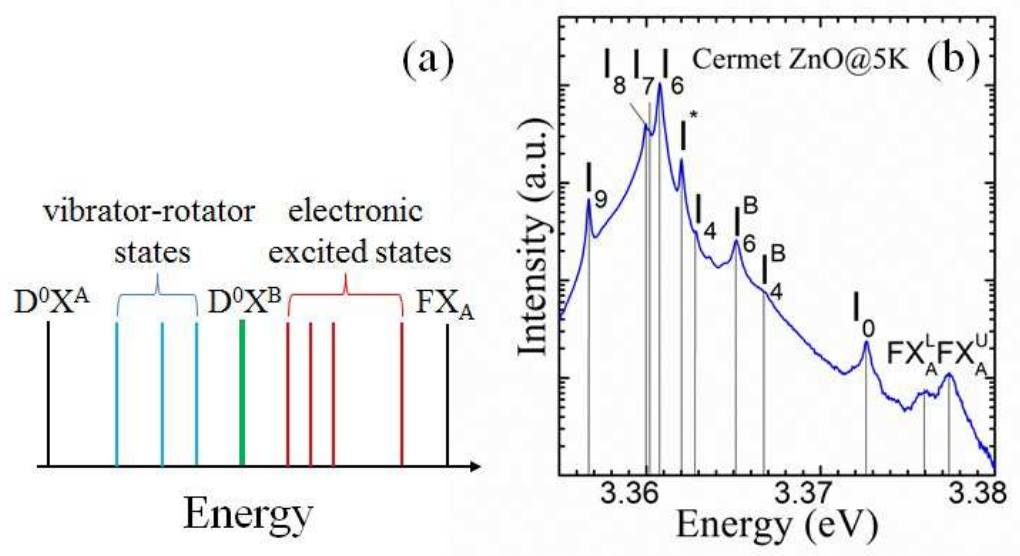

Figure 4.2 (a) Schematics illustrating energy levels of the BX ground and excited states. (b) A typical $5 \mathrm{~K}$ PL spectrum from bulk $\mathrm{ZnO}$. 
ground and excited states of DX transition are illustrated in Figure 4.2(a) (energies are not to scale).

Figure 4.2(b) shows a typical PL spectrum recorded within the near band-edge spectral range of bulk $\mathrm{ZnO}$ from Cermet Inc. The detected PL lines are labeled following the literature $[40,41]$ and are summarized in the following table.

\begin{tabular}{cccc}
\hline $\mathbf{B X}$ & $\mathbf{E}(\mathbf{e V})$ & $\mathbf{B X}$ & $\mathbf{E}(\mathbf{e V})$ \\
\hline $\mathbf{I}_{\mathbf{9}}\left(\mathrm{D}^{0} \mathrm{X}^{\mathrm{A}}\right)$ & 3.3567 & $\mathbf{I}_{\mathbf{4}}\left(\mathrm{D}^{0} \mathrm{X}^{\mathrm{A}}\right)$ & 3.3628 \\
$\mathbf{I}_{\mathbf{8}}\left(\mathrm{D}^{0} \mathrm{X}^{\mathrm{A}}\right)$ & 3.3598 & $\mathbf{I}_{\mathbf{6}}^{\boldsymbol{B}}\left(\mathrm{D}^{0} \mathrm{X}^{\mathrm{B}}\right)$ & 3.3652 \\
$\mathbf{I}_{\mathbf{7}}\left(\mathrm{D}^{0} \mathrm{X}^{\mathrm{A}}\right)$ & 3.3600 & $\mathbf{I}_{\mathbf{4}}^{\boldsymbol{B}}\left(\mathrm{D}^{0} \mathrm{X}^{\mathrm{B}}\right)$ & 3.3669 \\
$\mathbf{I}_{\mathbf{6}}\left(\mathrm{D}^{0} \mathrm{X}^{\mathrm{A}}\right)$ & 3.3608 & $\mathbf{I}_{\mathbf{0}}\left(\mathrm{D}^{+} \mathrm{X}^{\mathrm{A}}\right)$ & 3.3727 \\
$\mathbf{I}^{*}\left(\mathrm{I}_{\text {iso }} \mathrm{X}\right)$ & 3.3620 & & \\
\hline \multicolumn{4}{c}{ Table 4.1 Bound exciton transitions in bulk $\mathrm{ZnO}$}
\end{tabular}

It can be seen from the table that the majority of the detected BX lines are from different $\mathrm{D}^{0} \mathrm{X}^{\mathrm{A}}$. The $\mathrm{D}^{0} \mathrm{X}^{\mathrm{B}}$ transitions are usually detected around $4.5 \mathrm{meV}$ above their corresponding $\mathrm{D}^{0} \mathrm{X}^{\mathrm{A}}$ emissions. This energy difference is determined by the spin-orbit splitting between A and B valence subbands. PL emissions related to rotator-vibrator and electronic excited states of $\mathrm{D}^{0} \mathrm{X}^{\mathrm{A}}$ usually overlap with intense PL lines from the ground $\mathrm{D}^{0} \mathrm{X}^{\mathrm{A}}$ states, which make them hard to resolve. By employing PL excitation (PLE) measurements [40], the excited states associated with $\mathrm{D}^{0} \mathrm{X}^{\mathrm{A}}$ could be clearly identified. Relative intensities of $\mathrm{D}^{0} \mathrm{X}^{\mathrm{A}}$ and $\mathrm{D}^{+} \mathrm{X}^{\mathrm{A}}$ depend on the donor binding energy $E_{D}$ and a Fermi level position. When the donor level lies below the Fermi level, the majority of donors become neutral, which leads to a weaker emission from $\mathrm{D}^{+} \mathrm{X}^{\mathrm{A}}$. On the other hand, $E_{l o c}$ depends linearly on $E_{D}$ according to the Haynes rule [42]. When $E_{D}$ is smaller than a threshold value $(47 \mathrm{meV}$ in $\mathrm{ZnO}[43,44])$, the localization energy $E_{l o c}$ of $\mathrm{D}^{+} \mathrm{X}^{\mathrm{A}}$ becomes negative, which is physically unrealistic [44]. As for $\mathrm{D}^{+} \mathrm{X}^{\mathrm{B}}$, it cannot be observed in emission as it has a higher energy level than $\mathrm{FX}_{\mathrm{A}}$ which makes it energetically unstable. 


\subsection{Optical selection rules for donor bound excitons}

The electronic configurations of $\mathrm{D}^{0} \mathrm{X}$ and $\mathrm{D}^{+} \mathrm{X}$ are distinctly different. Even for $\mathrm{D}^{0} \mathrm{X}$, it is differentiated by $\mathrm{A}$ or $\mathrm{B}$ valence hole identity. This leads to different selection rules for the corresponding optical transitions.

The wavefunction of an A valence hole in $\mathrm{D}^{0} \mathrm{X}^{\mathrm{A}}$ involves $|x\rangle,|y\rangle$ and $|z\rangle$ orbital states. During radiative recombination with an electron carrying the $|s\rangle$ orbital, the dipole strength $\langle s|\nabla \cdot \vec{e}| i\rangle(i=x, y, z)$ is non-zero for all three directions in space (the z-axis is set along the c-axis). Therefore, the $\mathrm{D}^{0} \mathrm{X}^{\mathrm{A}}$ transition is

(a)
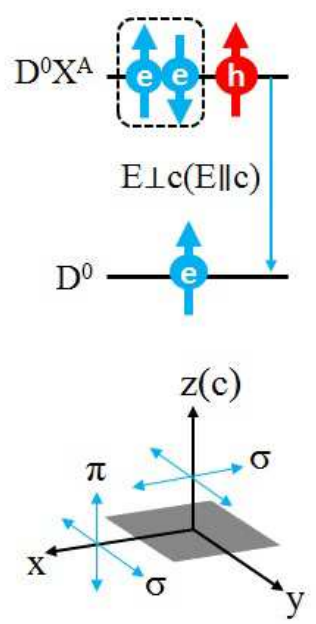

(b)
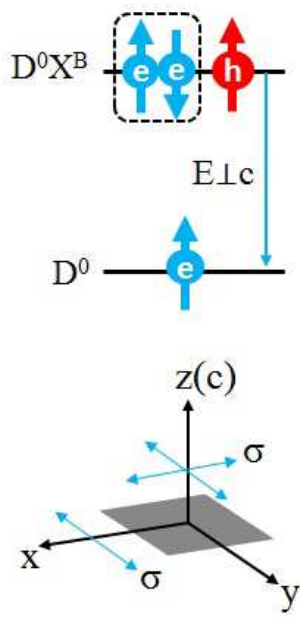

(c)
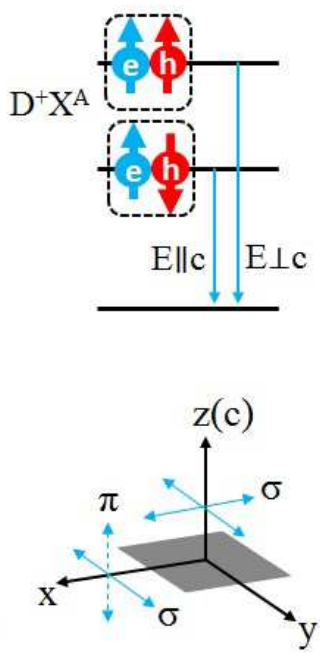

Figure 4.3 Schematic transition diagram and polarization geometry of the emissions for (a) $D^{0} X^{A}$, (b) $D^{0} X^{B}$, and (c) $D^{+} X^{A}$. The double arrow lines denote electric field vectors of the emissions, whereas the dotted arrow line stands for the emission of a different energy.

observable in both $\sigma(\mathrm{E} \perp \mathrm{c})$ and $\pi(\mathrm{E} \| \mathrm{c})$ polarization, which is illustrated in Figure 4.3(a). In contrast, the wavefunction of a $\mathrm{B}$-valence hole in $\mathrm{D}^{0} \mathrm{X}^{\mathrm{B}}$ only includes $|x\rangle$ and $|y\rangle$ orbitals, which results in polarization of the emission perpendicular to the c-axis. As is shown in Figure 4.3(b), only $\sigma$ polarization is allowed. The case of $\mathrm{D}^{+} \mathrm{X}^{\mathrm{A}}$ is a bit more complicated. Transitions from the upper $\Gamma_{5}$ exciton state are polarized perpendicular to the c-axis, i.e. give rise to the $\sigma$ - 
polarized emission. At the lower energy side, there are nearly degenerate $\Gamma_{1}$ and $\Gamma_{2}$ exciton levels. The $\Gamma_{1}$ exciton has a dipole along the c-axis that emits light in $\pi$ polarization as shown in Figure 4.3(c). However, its oscillator strength is much smaller as compared with the $\Gamma_{5}$ exciton. Optical transitions from the $\Gamma_{2}$ state are normally dipole-forbidden and cannot be detected.

These polarization properties could be used as a useful guideline to distinguish between the $\mathrm{D}^{0} \mathrm{X}^{\mathrm{A}}, \mathrm{D}^{0} \mathrm{X}^{\mathrm{B}}$ and $\mathrm{D}^{+} \mathrm{X}^{\mathrm{A}}$ lines. 


\section{Excitons in an external magnetic field}

By studying optical transitions of FX and BX under applied magnetic fields, it is possible to identify the nature of the binding centers (i.e. a donor, an acceptor or an isoelectronic center) and to determine their charge state. One can also obtain information about g-factors of the bound electron and hole. Moreover, in most cases, electrons and holes bound in FX and BX complexes are effective masslike and carry the character of conduction and valence band states. Therefore, splitting of the related transitions in an applied magnetic field reflects symmetries of the CB and VB states.

\subsection{Zeeman splitting of neutral donor and acceptor bound excitons}

Generally speaking, Zeeman splitting of an electronic state is induced by the interaction of an external magnetic field with a magnetic dipole moment associated with this state. $\mathrm{D}^{0} \mathrm{X}^{\mathrm{A}}$ has two electrons whose spins are antiparallel, resulting in a zero total magnetic dipole moment. Therefore it is only the A valence hole that will respond to the external magnetic field. The exciton state will then split into two Zeeman sublevels which correspond to the spin-down and spin-up states, as shown in Figure 5.1(a). The g-factor of A valence hole is rather anisotropic with $g_{h}^{\|}=-1.04$ and $g_{h}^{\perp}=0.2$. This anisotropy is also reflected by its wavefunction:

$$
\begin{aligned}
& \left|\Psi_{A}^{+}\right\rangle=i a|1,+1\rangle \Downarrow-i b|1,0\rangle \Uparrow \\
& \left|\Psi_{A}^{-}\right\rangle=a|1,-1\rangle \Uparrow+b|1,0\rangle \Downarrow(a \gg b)
\end{aligned}
$$

Here the in-plane component of the orbital angular momentum $|1, \pm 1\rangle$ is much more intense than the $|1,0\rangle$ component directed along the c-axis. An absolute value of the hole g-factor for an arbitrary direction of the magnetic field is given by 
(a)

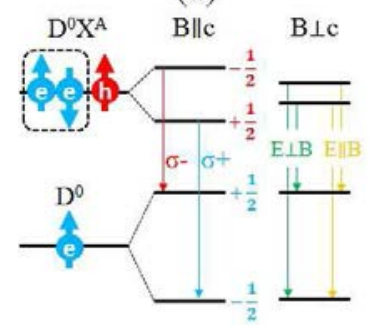

(c)

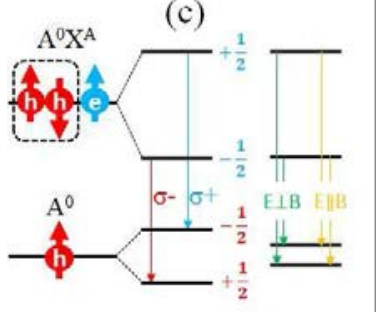

(b)

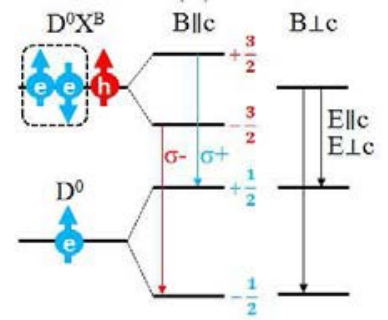

(d)

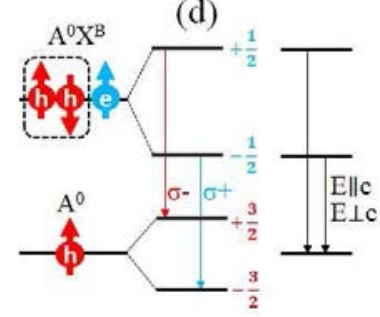

Figure 5.1 Zeeman splitting of the ground and excited state of $(a) D^{0} X^{A}$, (b) $D^{0} X^{B}$, (c) $A^{0} X^{A}$, and (d) $A^{0} X^{B}$ transition.

$$
\left|g_{h}\right|=\sqrt{\left(g_{h}^{\|} \cos \theta\right)^{2}+\left(g_{h}^{\perp} \sin \theta\right)^{2}} \quad(\theta=B \wedge c)
$$

where $\theta$ is the angle between a magnetic field and the c-axis. After the $\mathrm{D}^{0} \mathrm{X}^{\mathrm{A}}$ transition, a spin-unpaired electron is left at the donor site. The ground state (i.e. without BX) also splits into two Zeeman sublevels but the splitting is now solely determined by the electron g-factor $g_{e}$. Since the electron has an $|s\rangle$ orbital, the difference between $g_{e}^{\|}$and $g_{e}^{\perp}$ is quite small and usually could not be resolved in the magneto-photoluminescence. Thus $g_{e}$ could be regarded as isotropic. The $g_{e}$ value changes slightly among different shallow donors and is typically around $1.956[45,46]$.

When $\mathrm{B} \| \mathrm{c}$ and $\mathrm{k} \| \mathrm{c}$ (the Faraday configuration), two circularly polarized $\left(\sigma^{+}\right.$and $\sigma^{-}$) lines could be observed, which have energies

$$
E_{\sigma \pm}=E_{0} \pm \frac{1}{2}\left(g_{e}+g_{h}^{\|}\right) \mu_{B} B
$$

Here $E_{0}$ is the zero-field transition energy. When an applied magnetic field is rotated by an angle $\theta$ with respect to the c-axis, two additional Zeeman 
components emerge at higher and lower energy sides. The four Zeeman lines are now located at energies

$$
\begin{aligned}
& E^{ \pm}=E_{0} \pm \frac{1}{2}\left(g_{e}+\sqrt{\left(g_{h}^{\|} \cos \theta\right)^{2}+\left(g_{h}^{\perp} \sin \theta\right)^{2}}\right) \mu_{B} B \text { (the outer pair) } \\
& E^{ \pm}=E_{0} \pm \frac{1}{2}\left(g_{e}-\sqrt{\left(g_{h}^{\|} \cos \theta\right)^{2}+\left(g_{h}^{\perp} \sin \theta\right)^{2}}\right) \mu_{B} B \text { (the inner pair) }
\end{aligned}
$$

When $\mathrm{B} \perp \mathrm{c}$ and $\mathrm{k} \| \mathrm{c}$ (Voigt configuration), the outer pair of the Zeeman components become polarized perpendicular to the magnetic field $(\mathrm{E} \perp \mathrm{B})$ while the inner pair emit in the $\mathrm{E} \| \mathrm{B}$ polarization.

The electronic structure of $\mathrm{D}^{0} \mathrm{X}^{\mathrm{B}}$ is similar to that of $\mathrm{D}^{0} \mathrm{X}^{\mathrm{A}}$, but the Zeeman splitting pattern is different due to the fact that $g_{h}^{\|}>0$ and $g_{h}^{\perp}=0$ for the B valence holes. The vanishing $g_{h}^{\perp}$ is caused by a lack of the $|1,0\rangle$ orbital in the hole wavefunction. Energies of the Zeeman components when $\mathrm{B} \| \mathrm{c}$ and $\mathrm{k} \| \mathrm{c}$ are now

$$
E^{\sigma \pm}=E_{0} \mp \frac{1}{2}\left(g_{e}-g_{h}^{\|}\right) \mu_{B} B
$$

For an arbitrary angle $\theta$ between B and the c-axis, all four Zeeman lines can be observed and have the following energies:

$$
\begin{aligned}
& E^{ \pm}=E_{0} \pm \frac{1}{2}\left(g_{e}+g_{h}^{\|} \cos \theta\right) \mu_{B} B \text { (the outer pair) } \\
& E^{ \pm}=E_{0} \pm \frac{1}{2}\left(g_{e}-g_{h}^{\|} \cos \theta\right) \mu_{B} B \text { (the inner pair) }
\end{aligned}
$$

Due to $g_{h} \frac{1}{h}=0$, the Zeeman splitting becomes doublet when $\mathrm{B} \perp \mathrm{c}$ and is only determined by $g_{e}$. It should be noted that the Zeeman behavior of $\mathrm{D}^{0} \mathrm{X}^{\mathrm{B}}$ in $\mathrm{B} \perp \mathrm{c}$ is substantially different from that for $\mathrm{D}^{0} \mathrm{X}^{\mathrm{A}}$ which exhibits the quadruplet splitting. Therefore, this unique difference could be used to distinguish between $\mathrm{D}^{0} \mathrm{Xs}$ involving $\mathrm{A}$ and $\mathrm{B}$ valence holes.

The Zeeman behavior of $\mathrm{A}^{0} \mathrm{X}^{\mathrm{A}}$ and $\mathrm{A}^{0} \mathrm{X}^{\mathrm{B}}$ is exactly the same as those for $\mathrm{D}^{0} \mathrm{X}^{\mathrm{A}}$ and $\mathrm{D}^{0} \mathrm{X}^{\mathrm{B}}$, except that the electron g-value determines now splitting of the excited state whereas the ground state splits according to the hole g-value. Their transitions are illustrated in Figure 5.1(c-d). 


\subsection{Zeeman splitting of excitons bound to isoelectronic centers and ionized donors and acceptors.}

$\mathrm{D}^{+} \mathrm{X}^{\mathrm{A}}, \mathrm{A}^{-} \mathrm{X}^{\mathrm{A}}$ and $\mathrm{I}_{\mathrm{iso}} \mathrm{X}^{\mathrm{A}}$ all consist of one electron-hole pair in the complex. After the transition, the ground state is a singlet level without any particle. As is shown in Figure 5.2, when $\mathrm{B} \| \mathrm{c}$ and $\mathrm{k} \| \mathrm{c}$, the $\Gamma_{5}$ exciton states split into two Zeeman components emitting $\sigma^{+}$- and $\sigma^{-}$- polarized light with energies

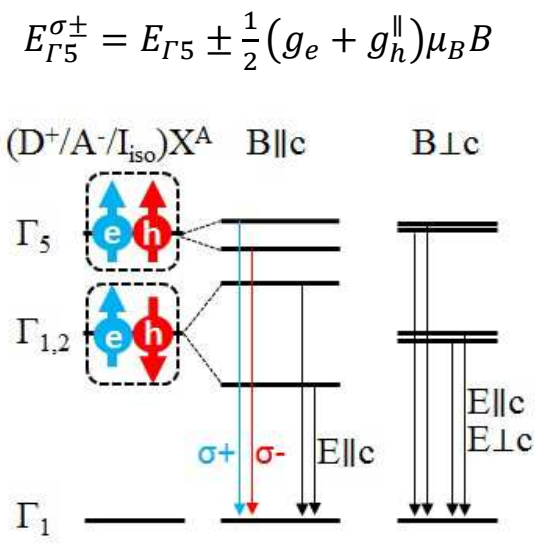

Figure 5.2 Schematic Zeeman splitting of $D^{+} X^{A}, A X^{A}$ and $I_{i s o} X^{A}$

Here $E_{\Gamma 5}, E_{\Gamma 1}$ and $E_{\Gamma 2}$ are the zero-field energies of the $\Gamma_{5}, \Gamma_{1}$ and $\Gamma_{2}$ exciton states. The lower $\Gamma_{1}$ and $\Gamma_{2}$ exciton states are mixed in a magnetic field and emit in $\pi$-polarization $(\mathrm{E} \| \mathrm{c})$ with

$$
E_{\Gamma 1,2}^{ \pm}=E_{\Gamma 2}+\frac{\Delta_{\Gamma 1,2}}{2} \pm \frac{1}{2} \sqrt{\Delta_{\Gamma 1,2}^{2}+\left[\left(g_{e}-g_{h}^{\|}\right) \mu_{B} B\right]^{2}}
$$

where $\Delta_{\Gamma 1,2}=E_{\Gamma 1}-E_{\Gamma 2} \approx 0[11,20]$.

In $\mathrm{B} \perp \mathrm{c}$, there is a coupling between the $\Gamma_{5}$ and $\Gamma_{1,2}$ exciton states and the coupling strength increases with increasing $B$. At weak magnetic fields, the upper energy state retains the $\Gamma_{5}$ character and the emission is predominantly $\sigma$ polarized $(\mathrm{E} \perp \mathrm{c})$ as admixture of the $\Gamma_{1}$ exciton state which emits in the $\pi$ polarization $(\mathrm{E} \| \mathrm{c})$ remains small. With increasing $\mathrm{B}$, the contribution of the $\Gamma_{1}$ component increases which leads to a decrease in the PL polarization. For the lower energy states, a weak magnetic field does not change the character of the 
$\Gamma_{1,2}$ components. With increasing magnetic fields, however, the $\Gamma_{5}$ exciton component becomes more intense which also depolarizes the emission. The energies of all four Zeeman states are derived as

$$
\begin{aligned}
& E_{\Gamma 5,1}^{ \pm}=E_{\Gamma 1}+\frac{\Delta_{\Gamma 5,1}}{2} \pm \frac{1}{2} \sqrt{\Delta_{\Gamma 5,1}^{2}+\left[\left(g_{e}+g_{h}^{\perp}\right) \mu_{B} B\right]^{2}} \\
& E_{\Gamma 5,2}^{ \pm}=E_{\Gamma 2}+\frac{\Delta_{\Gamma 5,2}}{2} \pm \frac{1}{2} \sqrt{\Delta_{\Gamma 5,2}^{2}+\left[\left(g_{e}-g_{h}^{\perp}\right) \mu_{B} B\right]^{2}}
\end{aligned}
$$

since $\Delta_{\Gamma 1,2} \approx 0, \Delta_{\Gamma 5,1}=E_{\Gamma 5}-E_{\Gamma 1} \approx E_{\Gamma 5}-E_{\Gamma 2}=\Delta_{\Gamma 5,2}$.

From the above discussion, it could be concluded that the Zeeman splitting is nonlinear when $\mathrm{B} \perp \mathrm{c}$, which is a distinctive fingerprint of this class of $\mathrm{BXs}$, in sharp contrast with the linear behavior of $\mathrm{D}^{0} \mathrm{Xs}$. 


\section{Exciton-phonon coupling}

\subsection{Phonons in semiconductors}

A semiconductor crystal could be viewed as a set of atoms attached to a periodic framework of lattice. In reality, the atoms never remain still at their lattice sites, but vibrate around their equilibrium positions. In the first order approximation, an interatomic potential could be considered as a harmonic potential. Thus an individual atom moves like a harmonic oscillator and all oscillating atoms form various modes of vibrational waves which carry energy and momentum. In the

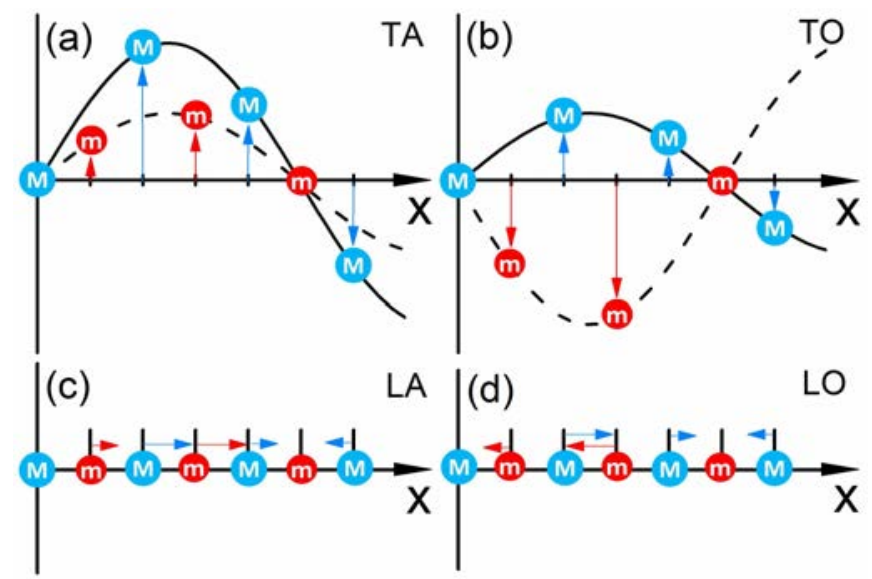

Figure 6.1 Atoms in a binary semiconductor vibrate in (a) transverse acoustical phonon (TA) mode, (b) transverse optical phonon (LO) mode, (c) longitudinal acoustical phonon (LA) mode and (d) longitudinal optical phonon (LO) mode.

language of quantum mechanics, the energy associated with each vibrational mode could be quantized and the corresponding quantum is called a phonon. A simple and qualitatively good model to describe the vibrational wave or phonon behavior is based on a one-dimensional crystal. Within this model, phonon modes in a binary compound semiconductor can be understood by considering vibration of atoms in a diatomic linear chain of two different elements. 
Shown in Figure 6.1 are allowed phonon modes propagating along a crystal. For acoustical phonon modes, atoms of different types are displaced in the same direction. On the contrary, the neighboring atoms oscillate in anti-phase for optical phonon modes. The propagating direction or wave vector of a phonon mode could be either perpendicular or along the direction for atoms displacement resulting in transverse or longitudinal phonons, respectively. In a realistic three dimensional semiconductor crystal with $n$ atoms in a primitive unit cell, all the atoms are allowed to move in three directions. This would give rise to a total of $3 n$ phonon modes, among which 3 modes belong to acoustical phonons and the other $(3 n-3)$ modes to optical phonons.

The distinct vibration patterns characteristic of each type of phonon modes perturb the periodic crystal lattice in different ways. As a consequence, this leads to various types of carrier-phonon scattering processes, among which the most important and common mechanisms are Fröhlich interaction and deformation potential interaction [47].The next section will elaborate on these interactions.

\subsection{Exciton-phonon coupling}

In the deformation potential interaction, both acoustical and optical phonons are involved in scattering of carriers. The phonon vibrations cause periodic modulation of interatomic distances which alters the bond length and bond angle between neighboring atoms. The crystal deformation of this kind is similar to applying strain on a crystal, which results in changes of conduction band and valence band energy in the Brillouin zone. As a consequence, free carriers and excitons sense this periodic perturbation of the energy bands which results in their scattering. The deformation potential interaction is independent of the phonon wave vector and is the dominant scattering mechanism in non-polar semiconductors like Si and Ge.

For the Fröhlich interaction, carrier scattering is induced by longitudinal-optical (LO) phonons. From Figure 6.1(d), in the LO-phonon mode, ion cores that carry opposite charges within the unit cell oscillate in anti-phase, which creates an internal electric field. This oscillating electric field acts as a perturbation which inelastically scatters free carriers and excitons. Therefore, the Fröhlich interaction of the LO-phonons with charge carriers or excitons is a Coulomb 
interaction and the interaction strength is dependent on the wave vector $\mathrm{k}$ of the LO-phonon. The Fröhlich interaction is the major mechanism responsible for the phonon scattering in polar semiconductors such as $\mathrm{ZnO}$ and $\mathrm{GaN}$ and will hence be further discussed hereafter.

The Hamiltonian that describes the exciton-LO-phonon coupling can be expressed as [48]:

$$
H_{e x-p h}=\sum_{m, m^{\prime}, k, q} V^{m m^{\prime}}(q) a_{m, k+q}^{+} a_{m^{\prime}, k}\left(b_{q}+b_{-q}^{+}\right)
$$

with

$$
V^{m m^{\prime}}=\sqrt{\frac{2 \pi e^{2} \hbar \omega_{L O}}{\Omega q^{2}}\left(\frac{1}{\varepsilon_{\infty}}-\frac{1}{\varepsilon_{0}}\right)} \int \phi_{m^{\prime}}^{*}(r) e^{i q \cdot r} \phi_{m}(r) d^{3} r
$$

Here $\hbar \omega_{L O}$ is the LO-phonon energy, $\Omega$ is the crystal volume, $\left(a^{+}, a\right)$ and $\left(b^{+}, b\right)$ are the creation and annihilation operators for the exciton and phonon, $\phi_{m}(r)$ denotes the exciton wavefunction with quantum number $m . V^{m m^{\prime}}$ is the matrix element for the exciton-LO-phonon coupling, $k$ and $q$ represent the wave vectors for exciton and phonon, respectively. One direct consequence of the coupling between the exciton and the LO-phonon is the appearance of phonon replicas of $\mathrm{FX}$ and BX emissions. Intensities of the phonon replica in different orders are related to the intensities of the zero-phonon $\mathrm{FX}$ and $\mathrm{BX}$ emissions as:

$$
\begin{gathered}
I_{n}=I_{0} \frac{S^{n}}{n !} n=0,1,2, \cdots \\
S=\sum_{q} \frac{|V(q)|^{2}}{\left(\hbar \omega_{L O}\right)^{2}}=\frac{2 \pi e^{2}}{\hbar \omega_{L O} \Omega}\left(\frac{1}{\varepsilon_{\infty}}-\frac{1}{\varepsilon_{0}}\right) \sum_{q} \frac{|f(q)|^{2}}{q^{2}} \\
f(q)=\int\left|\phi_{m}\right|^{2} e^{i q \cdot r} d^{3} r
\end{gathered}
$$

$S$ is the Huang-Rhys factor $[49,50]$ and $f(q)$ is the Fourier transformed relative distribution of the electron and hole wavefunctions in the exciton.

In the case of shallow $\mathrm{BX}$ in $\mathrm{ZnO}$, line-widths of the $\mathrm{BX}$-nLO emissions are usually comparable with that of the BX zero-phonon line, see Figure 6.2. This is because the involved bound carriers are effective mass-like and their wavefunctions are spread over the lattice. This results in a narrow BX distribution around the center of the Brillouin zone. Consequently, all of BXs 
may participate in both zero-phonon and phonon-assisted transitions. In the latter case, only phonons with wave vectors $q$ comparable to that of the $\mathrm{BX}$ wave vector $k$ will be involved. The strength of phonon replica scales with the intensity of the zero-phonon line. For example, a rise in temperature usually causes a gradual thermal dissociation of BX, resulting in quenching of the zerophonon and phonon-assisted transitions, as illustrated in Figure 6.2.

In the case of mobile FX, only a fraction of the FX population with translational wave vectors $k$ close to the center of the Brillouin zone will participate in the zero-phonon emission. However, all FX will be involved in the LO-phononassisted transitions since the nonzero k of mobile FX could be compensated by phonons. As a result, the FX-nLO replicas are broadened as compared with the zero-phonon FX line. This is illustrated in Figure 6.2 where positions of all FXrelated transitions are marked by the dotted lines. Intensities of the FX-nLOphonon replica can be expressed as [51,52]:

$$
I_{n}(E) \propto \sqrt{E} e^{-\frac{E}{k_{b} T}} W_{n}(E)
$$

This expression is simply the Maxwell-Boltzmann distribution of FX multiplied by a probability factor $W_{n}(E)$ for the FX with kinetic energy $E$ to couple with $n$

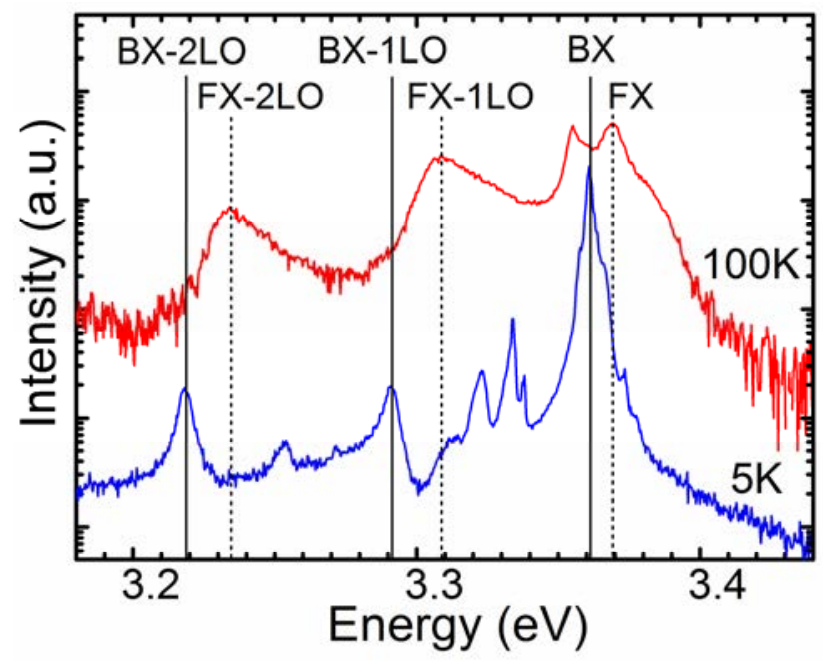

Figure 6.2 PL spectra of a Cermet $\mathrm{ZnO}$ bulk substrate measured at $5 \mathrm{~K}$ and $100 \mathrm{~K}$, respectively. The no-phonon $B X(F X)$ transition and its phonon replicas are marked by the solid (dotted) lines. 
LO-phonons. For the 1LO-phonon scattering process, $W_{1}(E)$ is proportional to $E$, whereas $W_{2}(E)$ for the 2LO-phonon scattering is independent of $E$ [27,47]. This independence of $E$ has essential experimental importance as the line shape of the 2nd phonon replica $I_{2}(E)$ generally reflects the population distribution of $\mathrm{FX}$ in the Brillouin zone.

To summarize, there are several important properties related to FX phonon replicas which can be derived from equation 6.6.

(1) The no-phonon FX emission is separated from the FX-1LO replica not by the LO phonon energy ( $72 \mathrm{meV}$ in $\mathrm{ZnO}$ ) but by $\mathrm{LO}-3 \mathrm{k}_{\mathrm{B}} \mathrm{T} / 2$, while the energy difference between $\mathrm{FX}$ and $\mathrm{FX}-2 \mathrm{LO}$ is $2 \mathrm{LO}-\mathrm{k}_{\mathrm{B}} \mathrm{T} / 2$.

(2) The line widths of the FX-1LO and FX-2LO transitions linearly scale with crystal temperature as $3 \mathrm{k}_{\mathrm{B}} \mathrm{T}$ and $2 \mathrm{k}_{\mathrm{B}} \mathrm{T}$, respectively.

(3) The ratio of spectrally integrated PL intensities between FX-1LO and FX$2 \mathrm{LO}$ is proportional to temperature $\mathrm{T}$. i.e.

$$
\frac{\int I_{F X-1 L O}(E) d E}{\int I_{F X-2 L O}(E) d E} \propto T
$$

where $I_{F X-n L O}(E)$ is the PL intensity of the corresponding LO-phonon replica. 


\section{Optical absorption}

\subsection{One-photon absorption}

Generally speaking, absorption is a reversed process with respect to light emission. For example, an electron residing in its ground state in the valence band or an electronic level within the bandgap can be optically promoted to an

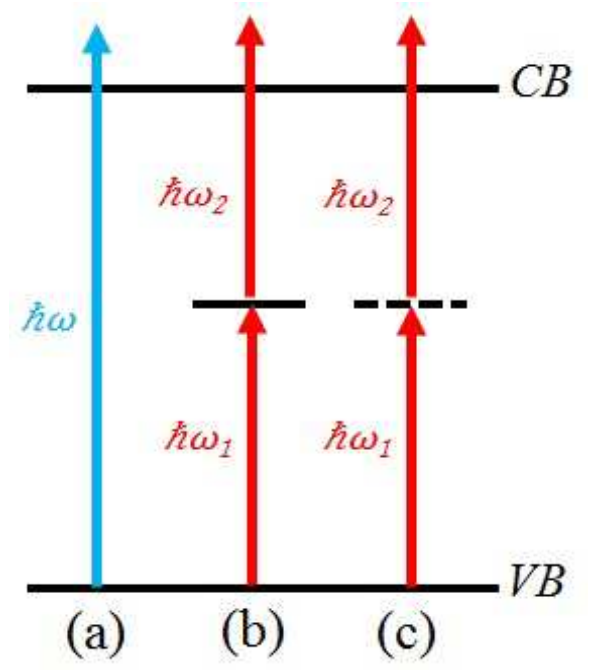

Figure 7.1 Transition scheme of (a) one-photon absorption, (b) two-step twophoton absorption via real intermediate state and (c) two-photon absorption via virtual state. The real and virtual states are represented by solid and dashed line, respectively.

excited state in the conduction band or an energetically higher level within the bandgap. During one-photon absorption (OPA) process, the energy and momentum differences between the initial ground and final excited states are conserved by the absorbed photon.

The one-photon absorption shown in Figure 7.1(a) is a linear optical process and can be quantum-mechanically described by the Fermi's golden rule [53]. 


$$
\begin{gathered}
W_{j i}=\frac{2 \pi}{\hbar}\left(\frac{e E_{0}}{m \omega}\right)^{2}|\langle j|\vec{e} \cdot \hat{p}| i\rangle|^{2} \delta\left(E_{j}-E_{i}-\hbar \omega\right) \\
\vec{e} \cdot \hat{p}=-i \hbar\left(e_{x} \frac{\partial}{\partial x}+e_{y} \frac{\partial}{\partial y}+e_{z} \frac{\partial}{\partial z}\right)
\end{gathered}
$$

Here $e$ and $\mathrm{m}$ are the charge and effective mass of the carrier, $E_{0}$ and $\omega$ represents the electric field and angular frequency of light, $|i\rangle$ and $|j\rangle$ denote the initial ground and final excited states which have energies $E_{i}$ and $E_{j}$, respectively. $\vec{e}$ is the unit vector representing light polarization, $\hat{p}$ is the momentum operator. For the case of band-to-band absorption process, the initial p-like orbital state in the valence band and the final s-like orbital state in the conduction band make the dipole matrix element $\langle j|\vec{e} \cdot \hat{p}| i\rangle$ non-zero, which indicates a dipole-allowed absorption process. Apart from the band-to-band transition, one-photon absorption also happens in a great variety of other optical processes as long as they are allowed from the dipole selection rule and the initial (final) state is filled (empty). Examples of such processes include transitions from the ground state to FX and BX levels, free-to-bound (or bound-to free) transitions i.e. $A^{-} \rightarrow A^{0}+$ $e(C B)$ and $D^{+}+e(V B) \rightarrow D^{0}$, etc.

\subsection{Two-photon absorption}

\subsubsection{Absorption via a real intermediate state}

The one photon absorption process cannot be accomplished if an incident photon energy is less than the energy difference between an initial and final state. However, if there is an intermediate state between them that act as an energy 'ladder', a charge carrier, e.g. an electron, now is able to absorb one photon to climb up to the intermediate state and to sequentially reach the final state via absorption of a second photon. This is the so-called two-step two-photon absorption (TS-TPA), see Figure 7.1(b). Different types of electronic states could act as an intermediate state, e.g. defect levels within the band gap [54-59], quantized electronic states in quantum structures [60-62], etc. Since two photons are required to complete the absorption process, its excitation power dependence 
is usually nonlinear. Efficiency of TS-TPA is closely dependent on the density and lifetime of the intermediate states.

\subsubsection{Absorption involving a virtual intermediate state}

Two-photon absorption can also occur via virtual intermediate states with two photons being absorbed simultaneously. This process is called two-photonabsorption (TPA) and is illustrated in Figure 7.1(c). The transition rate of the TPA process is expressed by the second order perturbation theory as $[27,63]$ :

$$
W_{j i}^{(2)}=\frac{2 \pi}{\hbar}\left(\frac{e E_{0}}{2 m \omega}\right)^{4}\left|\sum_{n} \frac{\langle f|\vec{e} \cdot \hat{p}| n\rangle\langle n|\vec{e} \cdot \hat{p}| i\rangle}{\hbar \omega-\left(E_{n}-E_{1}\right)}\right|^{2} \delta\left(E_{j}-E_{i}-2 \hbar \omega\right)
$$

The notations used here are the same as those in equation 7.1. $|i\rangle$ and $|j\rangle$ represent the real initial and final state, respectively. The two photons with same energy $\hbar \omega$ are absorbed via the virtual state denoted by $|n\rangle$. The TPA process is related to the third order optical susceptibility $\chi^{(3)}$ of the material, therefore it is a nonlinear optical effect and has quadratic dependence on the excitation light intensity $I_{\text {exc }}$ as shown from equation 7.3:

$$
W_{j i}^{(2)} \propto E_{0}^{4} \propto I_{e x c}^{2}
$$

Compared with one-photon and two-step two-photon absorption processes, an efficient TPA usually requires significantly higher power density of excitation.

By comparing equation 7.3 with equation 7.1 , it could be found that the dipole selection rule for the TPA absorption is different from the one-photon absorption case. This is because the absorption of one-photon changes the odd (even) parity of the initial state to even (odd) parity of the final state. On the other hand, in two-photon-absorption process, absorption of the second photon makes the parity of the final state the same as that of the initial state. Therefore, TPA could be used to excite carriers to those final states that are normally optically forbidden in the one-photon case. 


\section{Experimental techniques}

\subsection{Photoluminescence spectroscopy}

Photoluminescence (PL) spectroscopy is a commonly used method to characterize optical properties of semiconductor materials. Usually a laser beam with photon energy larger than the bandgap is used to excite a sample. The photon absorption results in creation of electrons in the conduction band and holes in the valence band (or free carrier continuum in the exciton picture). When the photo-excited electron and hole recombine radiatively, photons are emitted giving rise to $\mathrm{PL}$. In our low temperature $\mathrm{PL}$ study of $\mathrm{BX}$ in $\mathrm{ZnO}$, free carriers are created in continuum as shown in Figure 8.1(a), followed by the formation of FXs or BXs. The PL emitted from different bound states is then monitored. In the case of PL emission under an external magnetic field or magneto-

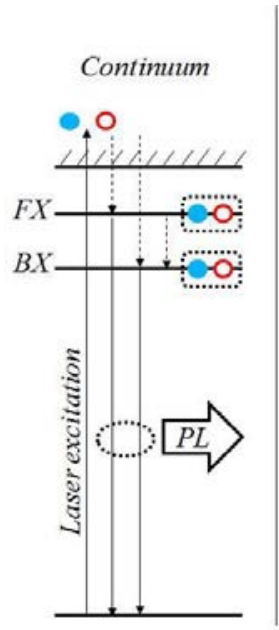

(a)

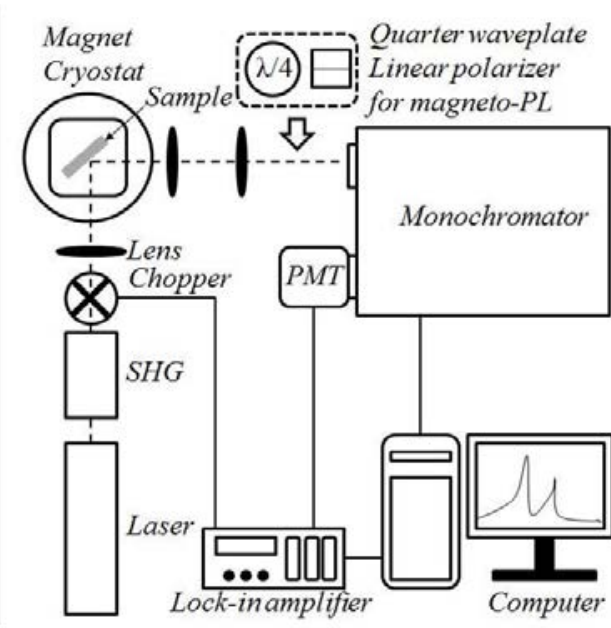

(b)

Figure 8.1 (a) A schematic diagram illustrating optical excitation of $F X$ and BX transitions. (b) Schematic of the experimental setup utilized for $c w-P L$ measurements.

photoluminescence, the originally spin degenerate $\mathrm{FX}$ and $\mathrm{BX}$ levels become split. Radiative transitions from the Zeeman sublevels can be polarized and light 
polarization depends on strength and orientation of the magnetic field. To resolve differently polarized emissions from the Zeeman sublevels, polarization optics such as a quarter waveplate and a linear polarizer are used.

Figure 8.1(b) shows a typical setup for the PL measurement. The excitation source is a 532-nm line from a solid-state continuous-wave (CW) laser, which is connected to a frequency doubling unit converting the wavelength of light to 266 $\mathrm{nm}$. After being modulated by a chopper, the $266-\mathrm{nm}$ laser beam is focused on a sample which is positioned inside a cryostat with a 10T superconducting magnet. The PL emission from the sample is collected by lenses and focused into a slit of a grating monochromator. At the end, the dispersed PL emission is registered by a photomultiplier (PMT) tube to convert light into an electrical signal.

\subsection{Time-resolved photoluminescence spectroscopy}

Time-resolved photoluminescence (TRPL) spectroscopy is an optical characterization method employed to study carrier dynamics. Pulsed laser excitation is used to create a non-equilibrium carrier population at an electronic level under the study. The excitation is restricted to a short time period (that was no longer than $3 \mathrm{ps}$ in our studies). Temporal evolution of the created population is then monitored by measuring the decay of the corresponding PL emission. This temporal evolution is jointly affected by different processes including radiative and competing non-radiative recombination, carrier transfer to/from other states, etc. Therefore, based on the PL dynamics, one could investigate various dynamic processes related to the electronic state of interest.

Shown in Figure 8.2 is a representative setup for TRPL measurements. The layout of the setup is similar to that for cw-PL measurements except that the excitation source now is a wavelength tunable Ti:Sapphire pulsed laser. On the detection side, a streak camera system is employed to record the time-dependent PL emission from the sample. 


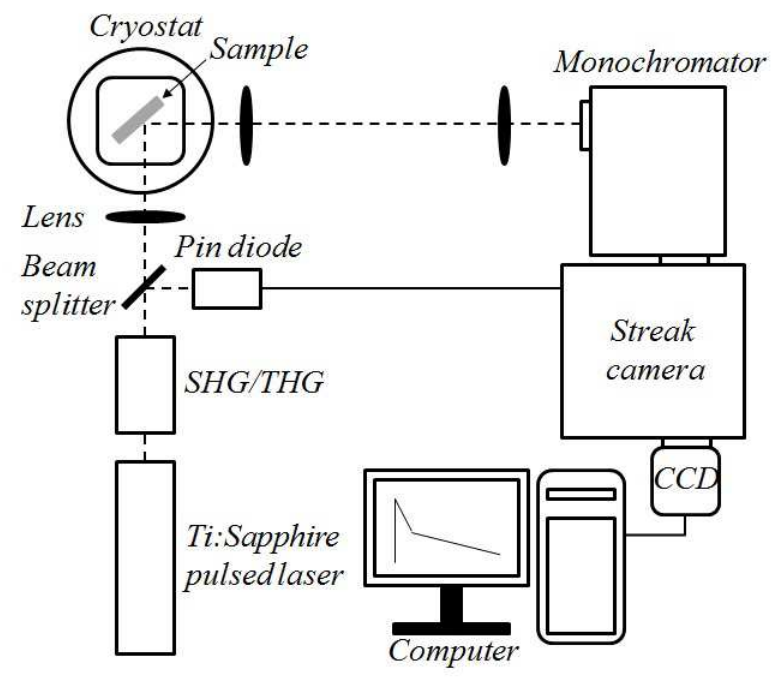

Figure 8.2 Schematic of a typical setup for time-resolved photoluminescence measurements.

\subsection{Time-resolved time-of-flight technique}

Time-of-flight (TOF) spectroscopy is commonly used to characterize light propagation though a medium. The principle of TOF is quite simple. A light pulse is sent into the medium at $t=0$, its propagation time $\Delta t$ inside the medium is counted. Knowing the thickness $d$ of the medium, the group velocity $V_{g}$ of light pulse can be calculated.

Shown in Figure 8.3 is a setup for the TOF measurements which employs a pulsed laser as a light source. Laser pulses are directed into the medium from the backside of the sample and are detected from its front side, i.e. in forward geometry. The transmitted laser pulses are temporally resolved by a streak camera system. 


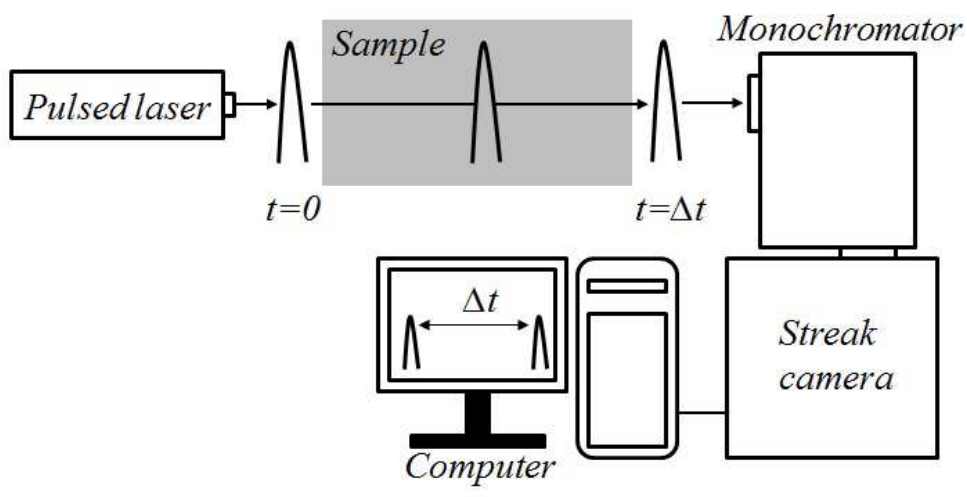

Figure 8.3 Schematic of a setup for time-of-flight measurements.

\subsection{Absorption spectroscopy}

Optical absorption is a reversed process as compared with photoluminescence. Whenever the incident photon energy fits the energy separation between an electronic ground and dipole-allowed excited state, the absorption process occurs.

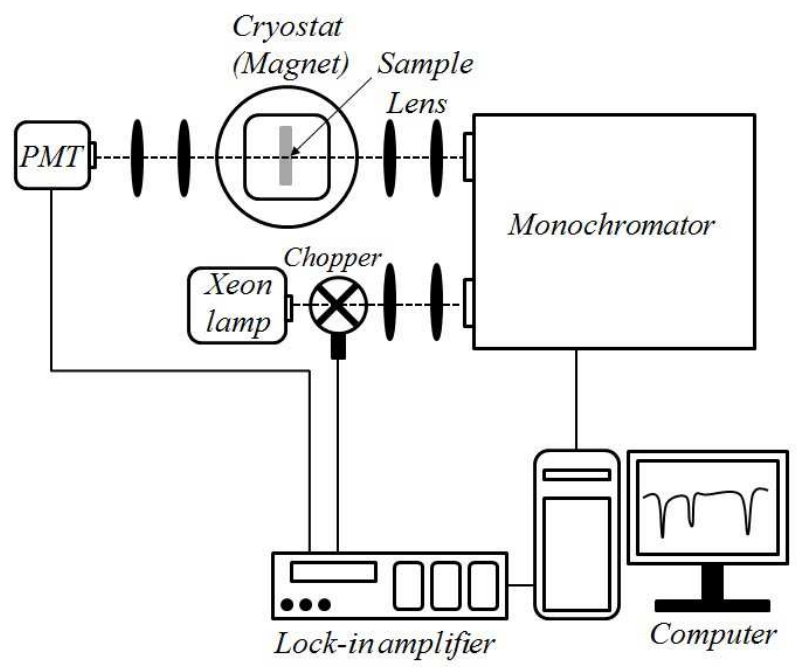

Figure 8.4 Schematic of a setup for absorption measurements. 
The light intensity under absorption decays exponentially as a function of the penetration depth. By detecting light transmitted through the sample at certain detection wavelengths, spectral dependence of the absorption coefficient associated with the monitored absorption process is measured.

The absorption spectroscopy is commonly used to identify excited states of an electronic system as the corresponding transitions are often quite weak in PL measurements. If performed at applied magnetic fields, it also allows one to clarify whether a ground electronic state involved in the absorption process is spin-degenerate.

Figure 8.4 shows a typical setup for one-photon absorption measurements. A Xenon lamp is used as an excitation source of which light is dispersed by a monochromator. The light transmitted through the sample is detected by a photodetector, e.g. a photomultiplier tube when the measurements are performed within the visible spectral range.

\subsection{Photoluminescence excitation spectroscopy}

If an electronic system has a set of excited states, only the excited state which is the lowest in energy usually gives rise to a detectable PL emission. Emissions related to the higher lying excited states are typically much weaker due to fast energy relaxation to the lower levels and are, therefore, not suitable for conventional PL studies. Photoluminescence excitation (PLE) spectroscopy is a technique that can overcome this problem. During the PLE measurements, the PL emission from the lowest excited state, for example BX at the ground state, is monitored while the excitation energy is continuously scanned through the higher excited states of the BX. This is shown schematically in Figure 8.5(a). Whenever the laser energy is in resonance with an excited state, the monitored PL emission will increase in intensity because of resonant photon absorption by the excited state followed by the fast relaxation of the photo-excited carriers to the lowest level. Therefore, by scanning the excitation energy through all energy levels, the excited states could be clearly identified and well-studied. 
In most cases, PLE is performed based on one-photon absorption. However, it is also possible to use two-photon absorption in PLE, i.e. two-photon PLE. Since the optical selection rules of two-photon PLE are different from those for the one-photon case, a set of excited states which are usually optically inactive in one-photon absorption process could be observed. This would provide additional information on the energy-level structure of the studied system.

A setup for two-photon PLE is shown in Figure 8.5(b). A cw wavelength-tunable Ti:Sapphire laser is used for excitation. All other components are the same as those utilized in the cw PL measurements discussed in section 8.1.

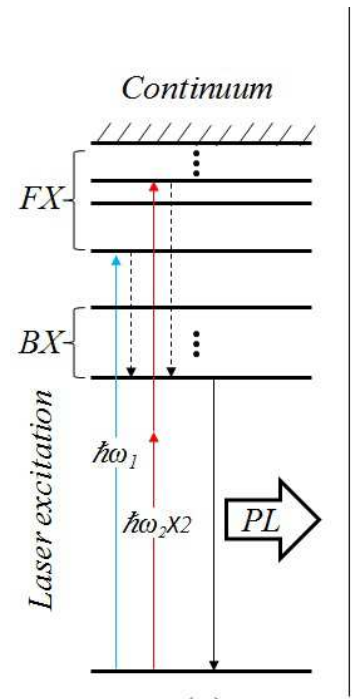

(a)

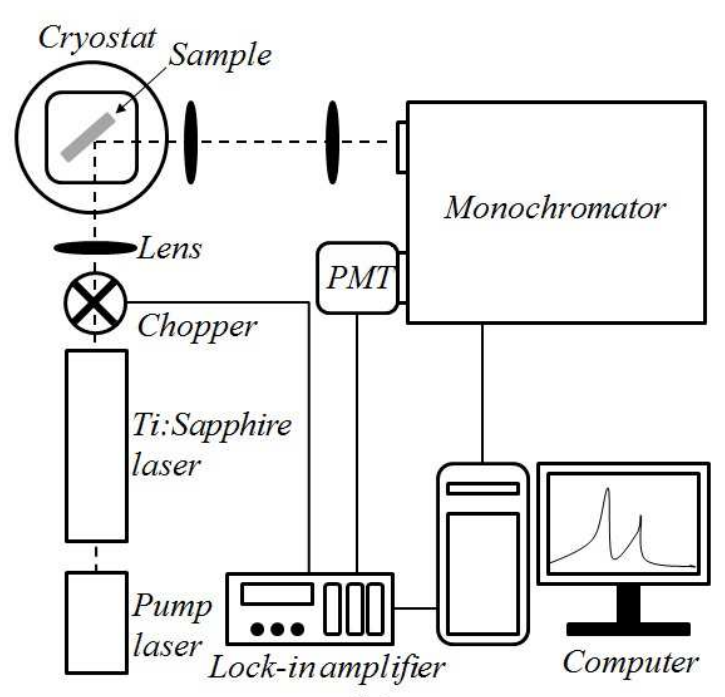

(b)

Figure 8.5 (a) Schematic transition diagram for one- and two-photon photoluminescence excitation. (b) Setup of two-photon photoluminescence excitation measurement.

\subsection{Cathodoluminescence spectroscopy}

Cathodoluminescence uses an electron beam instead of photons to excite a sample. A high-energy electron beam is created by an electron gun (usually a cathode ray tube) and is focused on a semiconductor sample in a similar manner like during optical excitation. Under electron bombardment, electrons in the 
valence band are excited to the conduction band, leaving behind holes. When the electrons and holes recombine radiatively, CL emission is emitted.

Figure 8.6 shows a setup for cathodoluminescence measurements. The excitation electron beam is generated by an electron gun and is focused on the sample. The CL emission is reflected to a monochromator by a mirror and finally recorded by a CCD.

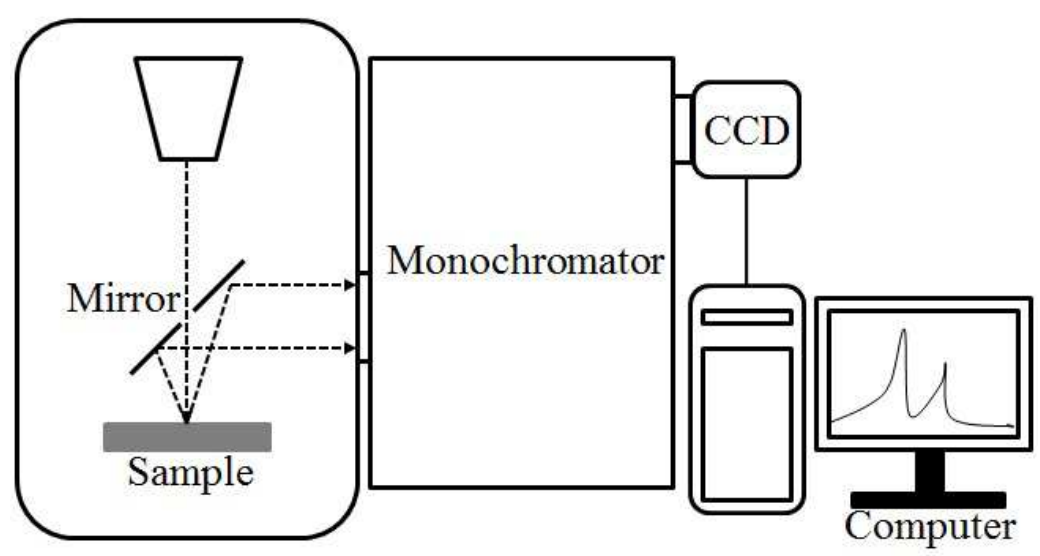

Figure 8.6 Schematic of a setup for cathodoluminescence measurements. 


\section{Summary of papers}

\section{Paper 1}

Time-resolved PL measurement was performed on $\mathrm{ZnO}$ tetrapods. PL decay from free excitons is observed to contain fast and slow components with time constants of 1 and $14 \mathrm{~ns}$ at room temperature. The fast PL decay arises from nonradiative recombination whereas the slow decay is suggested to reflect radiative lifetime of FX, from correlation between thermal increase of PL linewidth and FX lifetimes. The results indicate superior optical quality of the tetrapods since the decay time of the slow PL component is comparable to the longest lifetimes reported to date for $\mathrm{ZnO}$.

\section{Paper 2}

Time-resolved photoluminescence measurements were performed on shallow neutral donor bound excitons $\left(\mathrm{D}^{0} \mathrm{Xs}\right)$ in bulk $\mathrm{ZnO}$. It is found the PL decays of $\mathrm{D}^{0} \mathrm{X}$ transitions ( $\mathrm{I}_{6}-\mathrm{I}_{9}$ lines) are sensitive to excitation conditions, which change from a bi-exponential decay with fast $\left(\tau_{\mathrm{f}}\right)$ and slow $\left(\tau_{\mathrm{s}}\right)$ time constants under above-bandgap one-photon excitation to a single exponential decay with time constant $\tau_{\mathrm{s}}$ by two-photon excitation. The slow decay also dominates transients of longitudinal optical phonon-assisted and two-electron-satellite $\mathrm{D}^{0} \mathrm{X}$ transitions, and is attributed to "bulk" $\mathrm{D}^{0} \mathrm{X}$ lifetime. The fast component is tentatively attributed to the surface recombination.

\section{Paper 3}

Temperature dependent photoluminescence and cathodoluminescence measurements were performed to study free exciton emissions in $\mathrm{ZnO}$ tetrapods. The intensity of the no-phonon line is found to be weaker than longitudinal optical phonon assisted transitions, which is in sharp contrast to bulk ZnO. From spatially resolved CL measurement, this suppression of no-phonon line is shown 
to be affected by structural morphology of the $\mathrm{ZnO}$ tetrapods and becomes stronger in region with faceted surfaces. A model based on multiple internal reflection-induced reabsorption in the vicinity of the FX resonance is proposed to explain the observed effect.

\section{Paper 4}

By using time-of-flight spectroscopy, the group velocity of light propagating through bulk $\mathrm{ZnO}$ is shown to dramatically reduce down to $2044 \mathrm{~km} / \mathrm{s}$ when photon energy is in close vicinity of the absorption edge of the material. The slowing of light group velocity is found to depend on light polarization. It is concluded that although the slowdown is observed in the vicinity of donor bound exciton resonances, this effect is mainly determined by the free exciton polariton dispersion propagating via ballistic mode. From experimentally determined polariton group velocity, the polariton dispersion is accurately derived.

\section{Paper 5}

We used time-resolved PL and time-of-flight spectroscopy to study the propagation of exciton-polaritons through bulk $\mathrm{ZnO}$. When the photon energy is close to donor bound exciton resonances, substantial time delays of PL emission are observed which reach up to $210 \mathrm{ps}$ for a $0.55 \mathrm{~mm}$ thick crystal. By comparing results from time-of-flight measurements using PL light and laser pulses, the observed delay effect is found to arise from formation of exciton-polaritons and their spectral dispersion. It is also shown that the main contribution to the slowdown effect comes from free exciton-polaritons, while bound exciton-polaritons become important only in spectral region close to the corresponding resonances.

\section{Paper 6}

Time-resolved PL and magneto-PL measurements were performed on the socalled $\mathrm{I}_{6}^{\mathrm{B}}$ and $\mathrm{I}_{7}^{\mathrm{B}}$ transitions, which are previously assigned to neutral donor bound excitons involving a hole from the $\mathrm{B}$ valence band, $\mathrm{D}^{0} \mathrm{X}^{\mathrm{B}}$. It is found that 
PL transients of these transitions at $2 \mathrm{~K}$ are faster than those of their $\mathrm{I}_{6}$ and $\mathrm{I}_{7}$ counterparts with an A-VB hole. This is explained as being due to acoustic phonon-assisted energy relaxation of the hole. Based on magneto-PL measurements, effective g-factor values of conduction band electrons and B-VB holes are determined as $\mathrm{g}_{\mathrm{e}}=1.91, \mathrm{~g}_{h}^{\|}=1.79$, and $\mathrm{g}_{h}^{\perp}=0$, respectively.

\section{Paper 7}

Time-resolved PL and magneto-optical PL measurements were performed on a bound exciton transition at $3.3621 \mathrm{eV}$ (labeled as $\mathrm{I}^{*}$ ). Though the energy position of $I^{*}$ is within the same energy range as donor bound exciton transitions, its magneto-optical behavior is found to be substantially different from DXs bound to either ionized or neutral donors. A model based on exciton bound to an isoelectronic center with a hole-attractive local potential is shown to satisfactorily account for all experimental results of the $\mathrm{I}^{*}$ transition.

\section{Paper 8}

Efficient PL upconversion from donor-bound excitons is observed in bulk and nanorod $\mathrm{ZnO}$. Based on excitation power-dependent PL measurements performed at different excitation energies, two-photon absorption and two-step two-photon absorption processes are found to be responsible for the upconversion. The TS-TPA is found to occur via a defect/impurity (or defects/impurities) whose energy position lies within 1.14-1.56 eV from $\mathrm{CB}$ or VB edges. One of defect candidates responsible for TS-TPA could be $V_{Z n}$. An energy threshold which is distinct from that for the corresponding one-photon absorption is revealed for the TPA process and can be explained by selection rules for the involved optical transitions. 


\section{Bibliography}

1. Figure from www.espace.cern.ch/ISOLDE-SSP/SSP ISOLDE/ Photoluminescence Spectroscopy.aspx

2. T. Gruber, C. Kirchner, R. Kling, F. Reuss, and A. Waag, Appl. Phys. Lett. 84, 5359 (2004).

3. A. Ohtomo, M. Kawasaki, I. Ohkubo, H. Koinuma, T. Yasuda, and Y. Segawa, Appl. Phys. Lett. 75, 980 (1999).

4. X. Wang, C. J. Summers, and Z. L. Wang, Nano Lett. 4, 423 (2004).

5. Y. Fu, X. Du, S. A. Kulinich, J. Qiu, W. Qin, R. Li, J. Sun, and J. Liu, J. Am. Chem. Soc 129, 16029 (2007)

6. Z. L. Wang, J. Phys.: Condens. Matter 16, R829 (2004).

7. Z. L. Wang, Materialstoday 7, 26 (2004).

8. J. Dai, C. X. Xu, X. W. Sun, and X. H. Zhang, Appl. Phys. Lett. 98, 161110 (2011)

9. S. Chu, G. Wang, W. Zhou, Y. Lin, L. Chernyak, J. Zhao, J. Kong, L. Li, J. Ren, and J. Liu, Nature nanotechnology 6, 506 (2011)

10. Figure from www.whatiszincoxide.weebly.com/structure.html

11. W. R. L. Lambrecht, A. V. Rodina, S. Limpijumnong, B. Segall, and B. K. Meyer, Phys. Rev. B 65, 075207 (2002)

12. A. V. Rodina, M. Dietrich, A. Göldner, L. Eckey, A. Hoffmann, A. L. Efros, and M. Rosen, and B. K. Meyer, Phys. Rev. B 64, 115204 (2001)

13. D. C. Reynolds, C. W. Linton, and T. C. Collins, Phys. Rev. A 140, 1726 (1965)

14. D. G. Thomas, J. Phys. Chem. Solids 15, 86 (1960)

15. D. C. Reynolds, D. C. Look, and B. Jogai, C. W. Linton, G. Cantwell, and W. C. Harsch Phys. Rev. B 60, 2340 (1999) 
16. K. Hummer, Phys. Status Solidi B 86, 527 (1978)

17. P. Loose, M. Rosenzweig, and M. Wohleche, Phys. Status Solidi B 75, 137 (1976)

18. G. Blattner, C. Klingshirn, R. Helbig, and R. Meinl, Phys. Status Solidi B $107,105(1981)$

19. S. F. Chichibu, T. Sota, G. Cantwell, D. B. Eason, and C. W. Litton, J. Appl. Phys. 93, 756 (2003)

20. A. V. Rodina, M. Strassburg, M. Dworzak, U. Haboeck, A. Hoffmann, A. Zeuner, H. R. Alves, D. M. Hofmann, and B. K. Meyer, Phys. Rev. B 69, 125206 (2004).

21. M. R. Wagner, J. Schulze, R. Kirste, M. Cobet, A. Hoffmann, C. Rauch, B. K. Meyer, U. Röder, and K. Thonke, Phys. Rev. B 80, 205203 (2009).

22. D. W. Langer, R. N. Euwema, K. Era, and T. Koda, Phys. Rev. B 2, 4005 (1970)

23. B. Gil, and O. Briot, Phys. Rev. B 55, 2530 (1997)

24. M. Julier, J. Campo, B. Gil, J. P. Lascaray, and S. Nakamura, Phys. Rev. B 57, R6791

25. G. Blattner, G. Kurtze, G. Schmieder, and C. Klingshirn, Phys. Rev. B 25, 7413 (1982).

26. V. M. Agranovich, and V. L. Ginzburg, Crystal Optics with Spatial Dispersion, and Excitons

27. C. Klingshirn, Semiconductor Optics, Springer Berlin (2005).

28. J. Lagois, Phys. Rev. B 23, 5511 (1981).

29. J. Lagois, and K. Hummer, Phys. Stat. Sol. (b) 72, 393 (1975)

30. J. J. Hopfield, and D. G. Thomas, Phys. Rev. 132, 563 (1963) 
31. L. Sun, Z. Chen, Q. Ren, K. Yu, L. Bai, W. Zhou, H. Xiong, Z. Zhu, and X. Shen, Phys. Rev. Lett. 100, 156403 (2008)

32. L. K. Vugt, S. Ruhle, P. Ravindran, H. C. Gerritsen, L. Kuipers, and D. Vanmaekelbergh, Phys. Rev. Lett. 97, 147401 (2006)

33. K. Hummer, and P. Gebhardt, Phys. Stat. Sol. (b) 85, 271 (1978).

34. J. J. Hopfield, and D. G. Thomas, J. Phys. Chem. Sol. 12, 276 (1959).

35. R. L. Weiher, and W. C. Tait, Phys. Rev. 185, 1114 (1969).

36. C. Benoit, A. Guillaume, A. Bonnot, and J. M. Debever, Phys. Rev. Lett. 24, 1235 (1970).

37. K. Nassau, C. H. Henry, and J. W. Shiever, Proceedings of the 10th International Conference of Physics of Semiconductors, Cambridge, Massachusetts, 1970 (NBS, Springfield, VA 1970), P629.

38. J. L. Merz, H. Kukimoto, K. Nassau, and J. W. Shiever, Phys. Rev. B 6, 545 (1972)

39. B. Santic, C. Merz, U. Kaufmann, R. Niebuhr, H. Obloh, and K. Bachem, Appl.Phys. Lett. 71, 1837 (1997).

40. B. K. Meyer, J. Sann, S. Eisermann, and S. Lautenschlaeger, Phys. Rev. B 82, 115207 (2010).

41. B. K. Meyer, H. Alves, D. M. Hofmann, W. Kriegseis, D. Forster, F. Bertram, J. Christen, A. Hoffmann, M. Straßburg, M. Dworzak, U. Haboeck, A. V. Rodina, Phys. Stat. Sol. (b) 241, 231 (2004).

42. J. R. Haynes, Phys. Rev. Lett. 4, 361 (1960).

43.C. F. Klingshirn, B. K. Meyer, A. Waag, A. Hoffmann, and J. Geurts, Zinc Oxide: From fundamental properties towards novel applications, Springer Series in Materials Science, Springer Berlin (2010).

44. B. K. Meyer, J. Sann, S. Lautenschläger, M. R. Wagner, and A. Hoffmann, Phys. Rev. B 76, 184120 (2007). 
45. D. M. Hofmann, A. Hofstaetter, F. Leiter, H. Zhou, F. Henecker, B. K. Meyer, S. B. Orlinskii, J. Schmidt, and P. G. Baranov, Phys. Rev. Lett. 88, 045504 (2002).

46. D. Block, A. Herve, and R. T. Cox, Phys. Rev. B 25, 6049 (1982).

47. X. Zhang, T. Taliercio, S. Kolliakos, and P. Lefebvre, J. Phys.: Condensed Matter 13, 7053 (2001)

48. S. Rudin, T. L. Reinecke, and B. Segall, Phys. Rev. B 42, 11218 (1990)

49. K. Huang, and A. Rhys, Proc. R. Soc. A 204, 406 (1950)

50. A. Gurskii, and S. Viotikov, Sol. Stat. Comm. 112, 339 (1999)

51. B. Segall, and G. Mahan, Phys. Rev. 171, 935 (1968)

52. E. Gross, S. Permogorov, and B. Razbirin, J. Phys. Chem. Solids 27, 1647 (1966)

53. J. Davies, The physics of low dimensional semiconductors: an introduction, Cambridge University Press (1998).

54. W. Cao, W. Du, F, Su, and G. Li, Appl. Phys. Lett 89, 031902 (2006)

55. V. Ivanov, and Y. Semenov, M. Surma, and M. Godlewski, Phys. Rev. B 54, 4696 (1996)

56. Y. Rakovich, S. Filonovich, M. Gomes, J. Donegan, D. Talapin, A. Rogach, and A. Eychmuller, Phys. Stat. Sol. (b) 229, 449 (2002)

57. E. Poles, D. Selmarten, O. Micic, and A. Nozik, Appl. Phys. Lett. 75, 971 (1999)

58. A. Schindler, R. Bindemann, and K. Kreher, Phys. Stat. Sol. (b) 59, 439 (1973)

59. W. Chen, A. G. Joly, and D. E. McCready, J. Chem. Phys. 122, 224708 (2005) 
60. R. Hellmann, A. Euteneuer, S. Hense, J. Feldmann, P. Thomas, E. Göbel, D. Yakovlev, A. Waag, and G. Landwehr, Phys. Rev. B 51, 18053 (1995)

61. Y. Cho, D. Kim, B. Choe, H. Lim, J. Lee, and D. Kim, Phys. Rev. B 56, R4375 (1997)

62. P. P. Paskov, P. O. Holtz, B. Monemar, J. M. Garcia, W. V. Schoenfeld, and P. M. Petroff, Appl. Phys. Lett. 77, 812 (2000)

63. J. H. Bechtel, and W. L. Smith, Phys. Rev. B 13, 3515 (1976) 


\section{Part II}

\section{Papers}

The articles associated with this thesis have been removed for copyright reasons. For more details about these see:

http://urn.kb.se/resolve?urn=urn:nbn:se:liu:diva-102594 